\title{
Metastasis regulation by PPARD expression in cancer cells
}

\author{
Xiangsheng Zuo, ${ }^{1}$ Weiguo Xu, ${ }^{1,2}$ Min Xu, ${ }^{1}$ Rui Tian, ${ }^{1}$ Micheline J. Moussalli, ${ }^{3}$ Fei Mao, ${ }^{1}$ \\ Xiaofeng Zheng, ${ }^{4}$ Jing Wang, ${ }^{4}$ Jeffrey S. Morris, ${ }^{5}$ Mihai Gagea, ${ }^{6}$ Cathy Eng, ${ }^{1}$ Scott Kopetz, ${ }^{1}$ \\ Dipen M. Maru, ${ }^{3}$ Asif Rashid, ${ }^{3}$ Russell Broaddus, ${ }^{3}$ Daoyan Wei, ${ }^{7}$ Mien-Chie Hung, ${ }^{8}$ \\ Anil K. Sood, ${ }^{9,10,11}$ and Imad Shureiqi ${ }^{1}$ \\ 'Department of Gastrointestinal Medical Oncology, The University of Texas MD Anderson Cancer Center, Houston, Texas, \\ USA. ${ }^{2}$ Department of Surgical Oncology, Affiliated Hospital of Hebei United University, Tangshan, China. ${ }^{3}$ Department \\ of Pathology, ${ }^{4}$ Department of Bioinformatics and Computational Biology, ${ }^{5}$ Department of Biostatistics, ${ }^{6}$ Department of \\ Veterinary Medicine and Surgery, ${ }^{7}$ Department of Gastroenterology, ${ }^{8}$ Department of Molecular and Cellular Oncology, \\ ${ }^{9}$ Department of Gynecologic Oncology and Reproductive Medicine, and ${ }^{10}$ Department of Cancer Biology and "Center for \\ RNA Interference and Non-Coding RNAs, The University of Texas MD Anderson Cancer Center, Houston, Texas, USA.
}

Peroxisome proliferator-activated receptor- $\delta$ (PPARD) is upregulated in many major human cancers, but the role that its expression in cancer cells has in metastasis remains poorly understood. Here, we show that specific PPARD downregulation or genetic deletion of PPARD in cancer cells significantly repressed metastasis in various cancer models in vivo. Mechanistically, PPARD promoted angiogenesis via interleukin 8 in vivo and in vitro. Analysis of transcriptome profiling of HCT116 colon cancer cells with or without genetic deletion of PPARD and gene expression patterns in The Cancer Genome Atlas colorectal adenocarcinoma database identified novel prometastatic genes (CJA1, VIM, SPARC, STC1, SNCC) as PPARD targets. PPARD expression in cancer cells drastically affected epithelial-mesenchymal transition, migration, and invasion, further underscoring its necessity for metastasis. Clinically, high PPARD expression in various major human cancers (e.g., colorectal, lung, breast) was associated with significantly reduced metastasis-free survival. Our results demonstrate that PPARD, a druggable protein, is an important molecular target in metastatic cancer.

Conflict of interest: The authors have declared that no conflict of interest exists.

Submitted: October 24, 2016 Accepted: November 22, 2016 Published: January 12, 2017

Reference information: JCI Insight. 2017;2(1):e91419. doi:10.1172/jci.insight.91419.

\section{Introduction}

Metastasis remains a predominant cause of death in patients with cancers for which current treatments are generally non-curative. The progression of cancer cells to a metastatic state involves many molecular changes; however, the critical changes driving metastasis remain undefined (1-3).

Peroxisome proliferator-activated receptor $-\delta$ (PPARD) is a nuclear transcriptional receptor that regulates many molecular processes, including ones that potentially influence diseases such as cancer (4). PPARD is upregulated in various major human cancers, including colorectal, pancreatic, and lung cancer (5-8). Increased PPARD expression in cancer is associated with advanced pathological stage (7), which suggests that PPARD upregulation contributes to tumor progression. However, the role of PPARD in tumorigenesis and especially metastasis is poorly defined and often contested $(4,9)$.

Conflicting data have fueled the controversy regarding PPARD's role in tumorigenesis. For example, PPARD germline deletion increased intestinal tumorigenesis in $\mathrm{APC}^{\mathrm{Min}}$ mice in one study (10) but inhibited it in another (11). Others reported that the PPARD agonist GW501516 reduced pancreatic cell invasion in vitro despite PPARD being upregulated in human pancreatic ductal carcinoma (12). PPARD has also been reported to both promote $(11,13-15)$ and inhibit (16) angiogenesis, a mechanism critical to metastasis $(17,18)$. Although PPARD KO was initially reported to increase colonic tumorigenesis in one of the germline PPARD KO mouse models (10), later studies reported that PPARD KO instead inhibited tumorigenesis and angiogenesis when these mice were subcutaneously implanted with syngeneic B16 melanoma or Lewis lung carcinoma (LLC) cells $(7,19)$. These contradictory findings in the same mouse model have been interpreted as suggesting that PPARD has different roles depending on where it is expressed - specifically, that PPARD expressed in non-cancer cells promotes tumorigenesis, whereas PPARD expressed in 
tumor cells suppresses tumorigenesis $(7,19)$. However, these previous studies lacked experiments to assess whether specific PPARD expression modulation in cancer cells influences tumorigenesis. Furthermore, although some studies reported on PPARD expression affecting metastasis-related cellular events in vitro (20-22), the role of PPARD expression in cancer cells on metastasis remains to be defined in representative in vivo models. We therefore performed in-depth studies of PPARD using various experimental metastasis models and data from large patient cohorts to address this knowledge gap. Our results demonstrate that PPARD expression in cancer cells is a critical driver of metastasis.

\section{Results}

PPARD expression in cancer cells is critical to metastasis formation. To determine the effects that PPARD expression in cancer cells has on metastasis, we first generated B16-F10 cell lines stably transfected with PPARD-shRNA-A (PPARD-shRNA-A-clone1 and -clone2) and LLC-GFP cell lines (LLC cells GFP) stably transfected with a different PPARD-shRNA sequence (PPARD-shRNA-B). PPARD-shRNA-A transfection into B16-F10 cells and PPARD-shRNA-B into LLC-GFP cells significantly reduced PPARD mRNA and protein expression (Supplemental Figure 1, A-D; supplemental material available online with this article; doi:10.1172/jci.insight.91419DS1). Next, we used an experimental mouse model of blood-borne metastasis by tail vein injection to assess the effect of PPARD downregulation on metastasis. PPARD downregulation significantly inhibited the formation of lung metastases from both B16-F10 clones (Figure 1, A and B). Similar results were observed in a repeat experiment with B16-F10 PPARD-shRNA-A-clone1 and -clone2 (Figure 1, C and D). PPARD mRNA expression was significantly reduced in the lung metastases formed by PPARD-shRNA-A-clone1 or PPARD-shRNA-A-clone2 B16-F10 cells compared with the lung metastases formed by control-shRNA B16-F10 cells (Supplemental Figure 1E). The formation of lung metastases was confirmed histologically (Supplemental Figure 1F). We also transfected B16-F10 cells with different PPARD shRNA sequences using a lentivirus-based approach to confirm that these results were not specific to the shRNA sequence or method of shRNA transduction. PPARD downregulation by either PPARD-shRNA-C or -D significantly reduced PPARD expression (Supplemental Figure 1, G and H) and lung metastasis formation (Figure 1, E and F).

Consistent with the results in B16-F10 cells, PPARD downregulation by PPARD-shRNA-B into LLCGFP cells significantly inhibited lung metastases from LLC-GFP cells injected into tail veins (Figure 2, A and B). Similarly, lentivirus transduction of PPARD-shRNA-C and -D significantly reduced PPARD expression (Supplemental Figure 1, I and J) in LLC cells and LLC lung metastases (Figure 2, C and D).

To determine the extent to which PPARD expression in cancer cells is necessary for metastasis formation, we intravenously injected HCT116 colon cancer cells with genetic deletion of PPARD (PPARD-KO) into the tail veins of immunodeficient mice and assessed lung metastasis. PPARD-KO is a well-characterized cancer cell model generated by the genetic deletion via homologous recombination of the whole DNA binding domain sequence (exons 4-6) of PPARD (23). To further determine the specific role of PPARD, we reconstituted PPARD in PPARD-KO cells via stable PPARD plasmid transfection (PPARD-KO-PD cells). For a control, we stably transfected PPARD-KO cells with the control empty vector (PPARD-KO-C cells) (Supplemental Figure 2, A and B). Lung metastases formed in 8 of the 18 mice (44\%) injected with parental HCT116 cells, 0 of the 17 mice injected with PPARD-KO cells, 5 of the 15 mice (33\%) injected with PPARD-KO-PD cells, and 0 of the 15 mice injected with PPARD-KO-C cells (Figure 3A). The numbers of metastases per mouse for the groups injected with PPARD-KO or PPARD-KO-C cells were significantly different from HCT116 cells or PPARD-KO-PD cells (Figure 3B). The formation of lung metastases was confirmed histologically (Supplemental Figure 2C).

We then sought to determine whether ligand modulation of PPARD activity affects metastasis. HCT-116 parental cells stably transfected with the luciferase reporter gene generated by lentiviral gene transduction (HCT116-Luc cells) or HCT116 PPARD-KO cells were injected into the spleens of nude mice (24). The mice were fed identical diets with or without the PPARD agonist GW0742 $(1 \mathrm{mg} / \mathrm{kg})$. Mice fed the GW0742-containing diet had significantly more hepatic metastases than mice fed the control diet did (Figure 3, C-E), whereas PPARD$\mathrm{KO}$ cells injected into mice ( $n=11$ ) failed to form metastases (Figure 3, D and E).

To determine whether our results depended on the metastasis experimental modeling or growth of primary tumors, we used two independent orthotopic mouse models (Panc-02 pancreatic and 4T1 breast spontaneous metastasis models) to assess the effects that PPARD expression in cancer cells has on spontaneous metastasis. Panc-02 cells injected orthotopically into the pancreases of syngeneic C57BL/6 (B6) 
A

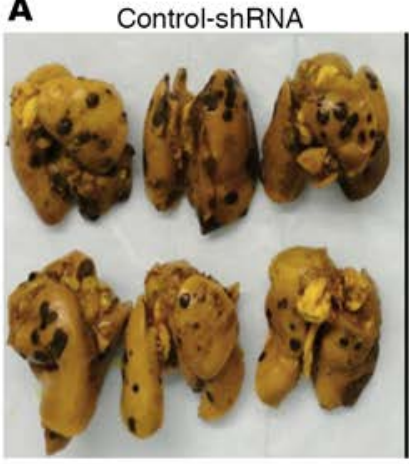

PPARD-shRNA-A-clone1

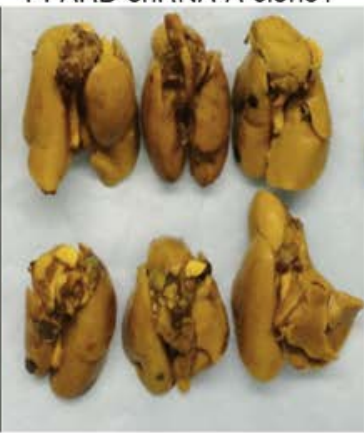

PPARD-shRNA-A-clone2

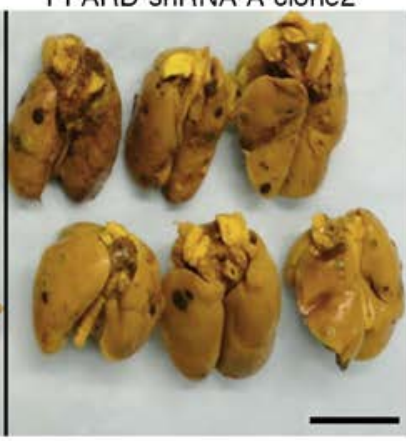

B

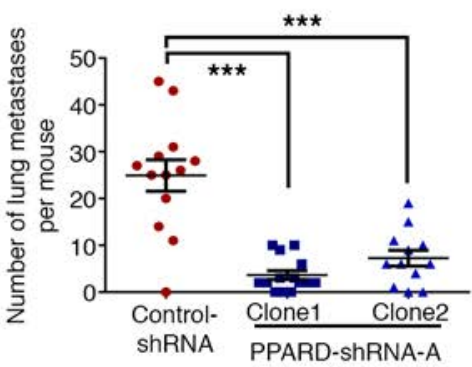

$\mathbf{F}$
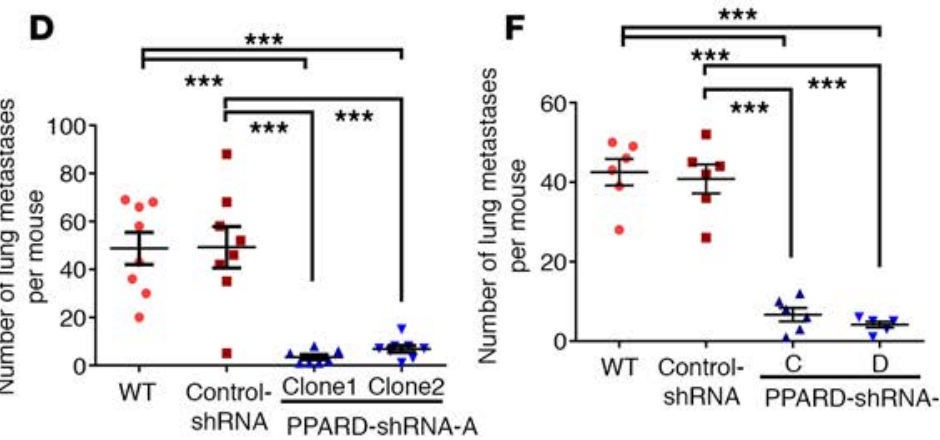

E

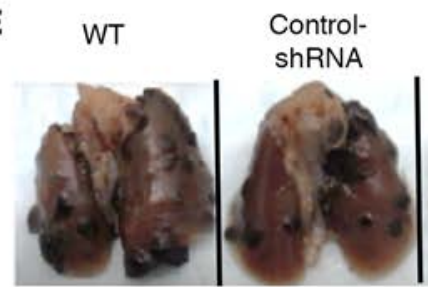

PPARD-shRNA-

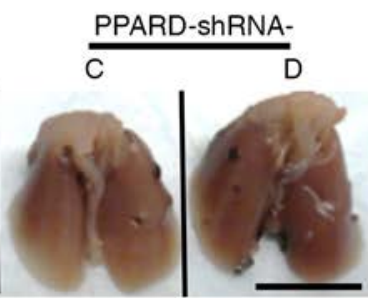

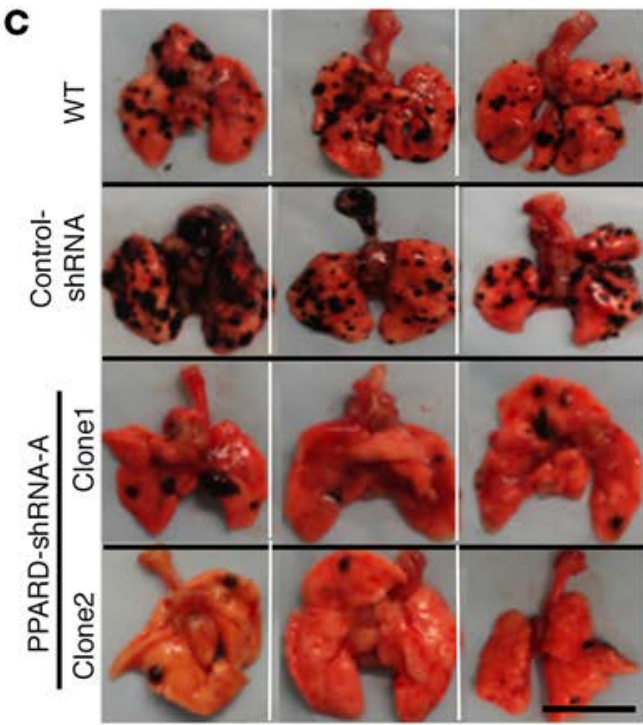

Figure 1. PPARD promotes lung metastases of B16-F10 melanoma cells in immunocompetent mice. (A-F) WT B16-F10 melanoma cells or B16-F10 melanoma cells stably transduced with PPARD-shRNA-A (PPARD-shRNXA-A-clone1 or -clone2) or control-shRNA plasmid, or with two independent PPARD-shRNA (PPARD-shRNA-C or -D) or control-shRNA lentivirus, were injected via the tail vein into syngeneic C57BL/6 mice, and lung metastasis formation was evaluated 3 weeks later. (A) Representative photographs of Bouin-fixed lung specimens of mice injected with plasmid-transfected cells. (B) Numbers of lung metastases per mouse for each group ( $n=12-14$ per group) shown in A. (C) Representative photographs of freshly isolated lungs in a repeat experiment. (D) Numbers of lung metastases per mouse for each group ( $n=8$ per group) shown in C. (E) Representative photographs of formalin-fixed lung specimens of mice injected with lentivirus-tranduced cells. (F) Numbers of lung metastases per mouse for each group ( $n=6$ per group) shown in $\mathbf{E}$. All scale bars: $1 \mathrm{~cm}$. Values in $\mathbf{B}, \mathbf{D}$, and $\mathbf{F}$ are mean $\pm \mathrm{SEM}$. Lines indicate means. ${ }^{* * *} P<0.0001$ (2-sided Poisson).

mice can form hepatic metastases (25), and $4 \mathrm{~T} 1$ breast cancer cells injected orthotopically into the mammary fat pads of syngeneic BALB/c female mice can form lung metastases $(26,27)$. Panc-02 cells stably transfected with PPARD-shRNA-A (PPARD-shRNA-A-clone1 or -clone2) had significantly lower PPARD RNA and protein expression than did WT Panc-02 cells and Panc-02 cells transfected with control-shRNA (Supplemental Figure 3, A and B). The pancreatic tumors in mice injected with PPARD-shRNA-A-clone1 or -clone2 weighed less than did those in mice injected with WT Panc-02 cells or control-shRNA Panc-02 cells (Figure 4, A and C). More strikingly, none of the 16 mice injected with Panc-02 cells transfected with PPARD-shRNA-A-clone1 or -clone2 had liver metastases (Figure 4, B and D). In contrast, histologically confirmed liver metastases (Supplemental Figure 3C) developed in 3 of the 8 mice injected with WT Panc02 cells and 3 of the 7 mice injected with control-shRNA Panc-02 cells; the numbers of hepatic metastases in mice injected with WT Panc-02 cells (mean \pm SEM: $0.90 \pm 0.44$ tumor/mouse) or control-shRNA Panc02 cells (mean \pm SEM: $0.90 \pm 0.46$ tumor/mouse) were not significantly different (Figure 4D).

$4 \mathrm{~T} 1$ cells stably transfected with two independent PPARD-shRNA-C or -D lentiviruses had significantly lower PPARD RNA and protein expression than did WT 4T1 cells and 4T1 cells transfected with control-shRNA (Supplemental Figure 3, D and E). For every mouse, the primary mammary tumor was 
A

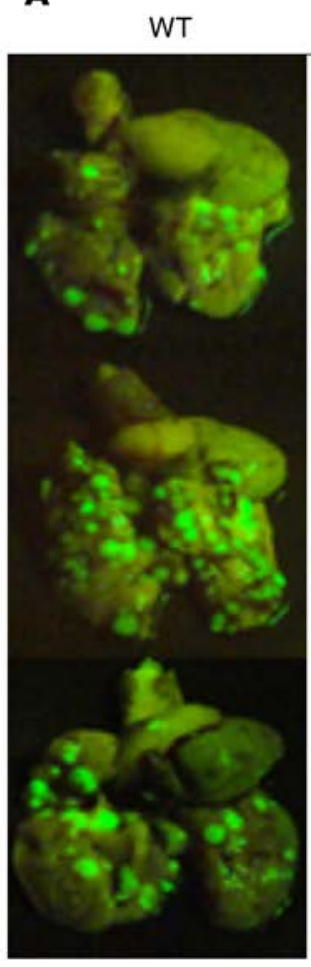

Control-shRNA

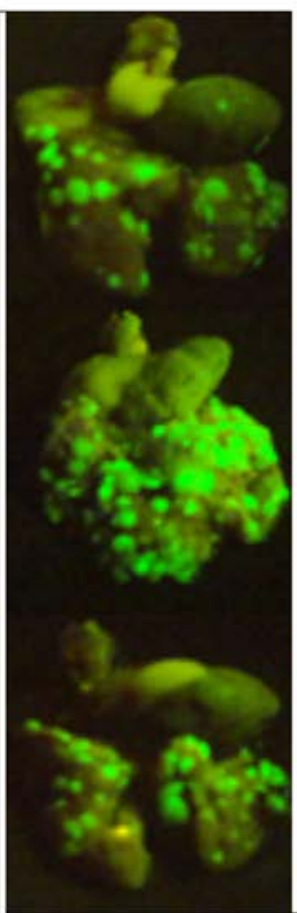

PPARD-shRNA-B

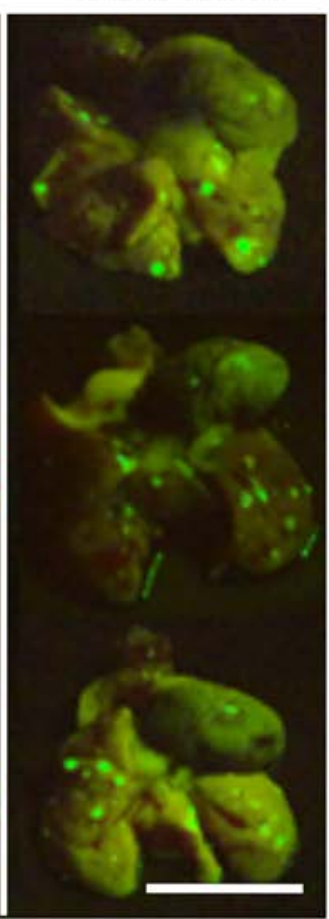

\section{B}
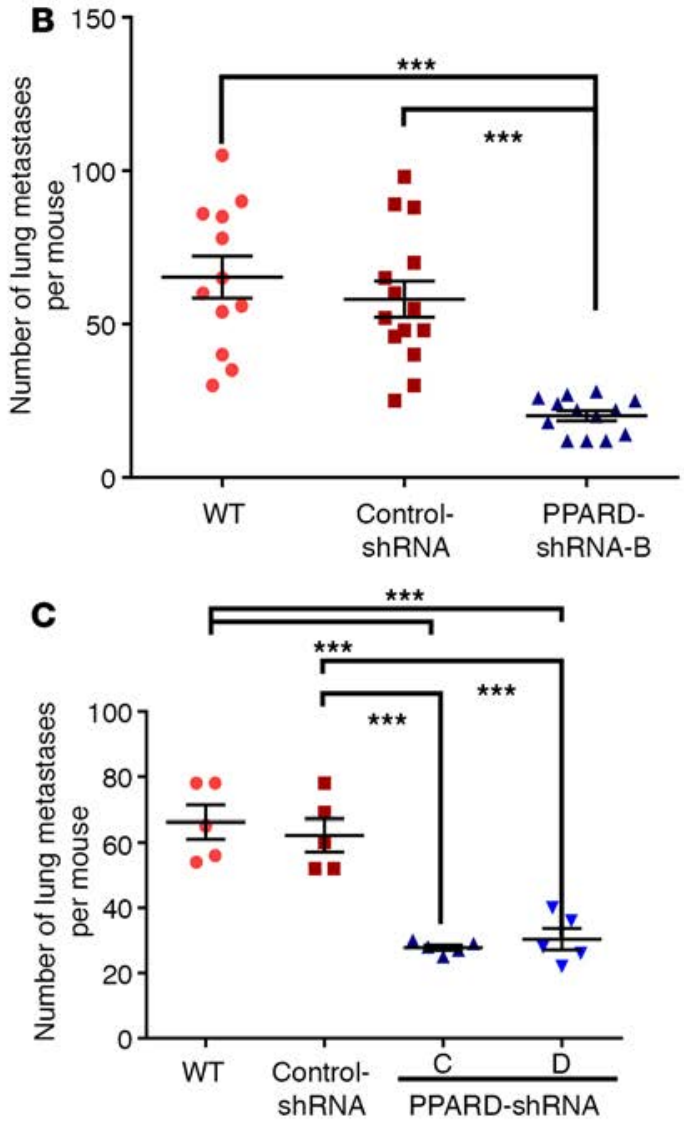

D WT

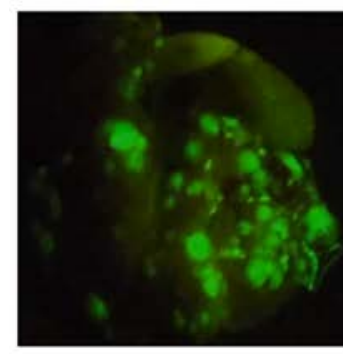

Control-shRNA

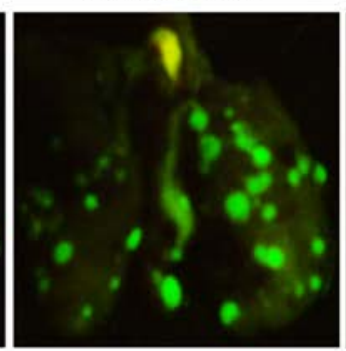

PPARD-shRNA

C

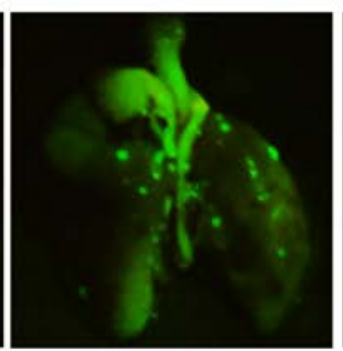

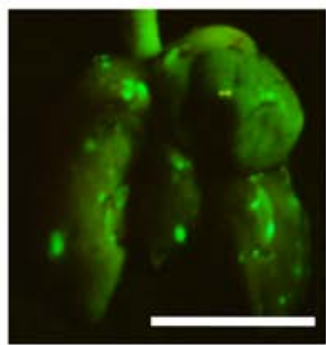

Figure 2. PPARD promotes LLC cells' formation of lung metastases in immunocompetent mice. (A-D) LLC-CFP WT cells or cells stably transduced with PPARD-shRNA-B or control-shRNA plasmid, or with two independent PPARD-shRNAs (PPARD-shRNA-C or -D) or control-shRNA lentivirus, were injected via the tail vein into syngeneic C57BL/6 mice, and lung metastasis formation was evaluated 2 weeks later. (A and D) Representative photographs of GFP fluorescence in isolated lungs from mice injected with plasmid-transfected cells (A) or lentivirus-transduced cells (D). (B and C) Numbers of lung metastases per mouse for each group shown in $\mathbf{A}(n=12-14$ per group) and $\mathbf{D}(n=5$ per group). Scale bars: $1 \mathrm{~cm}$. Values in $\mathbf{B}$ and $\mathbf{C}$ are mean \pm SEM. Lines indicate means. ${ }^{* *} P<0.0001$ (2-sided Poisson).

removed when it reached a predetermined size of $10 \mathrm{~mm}$ in the greatest diameter (Figure 4, E and $\mathrm{G}$ ). Two weeks after primary tumor removal, mice were examined for lung metastases with lung CT imaging followed by necropsies the next day. Metastasis multiplicity was significantly lower in mice injected with PPARD-shRNA-C cells (mean \pm SEM: $0.83 \pm 0.40$ tumor/mouse) or PPARD-shRNA-D cells $(1.33 \pm$ $0.49)$ than in mice injected with control-shRNA $4 \mathrm{~T} 1$ cells $(8.67 \pm 1.86)$ or WT $4 \mathrm{~T} 1$ cells $(9.00 \pm 1.37)$ (Figure 4, $\mathrm{F}$ and $\mathrm{H}$ ). Together, these results demonstrate that the modulation of PPARD expression in cancer cells has a profound effect on metastasis independent of experimental modeling.

PPARD expression in cancer cells is more important to metastasis than PPARD expression in non-cancer cells. Given the important role that tumor-host interaction has in tumor progression and metastasis, we directly compared the relative impact of PPARD expression in cancer cells versus non-cancer host cells on metas- 
A

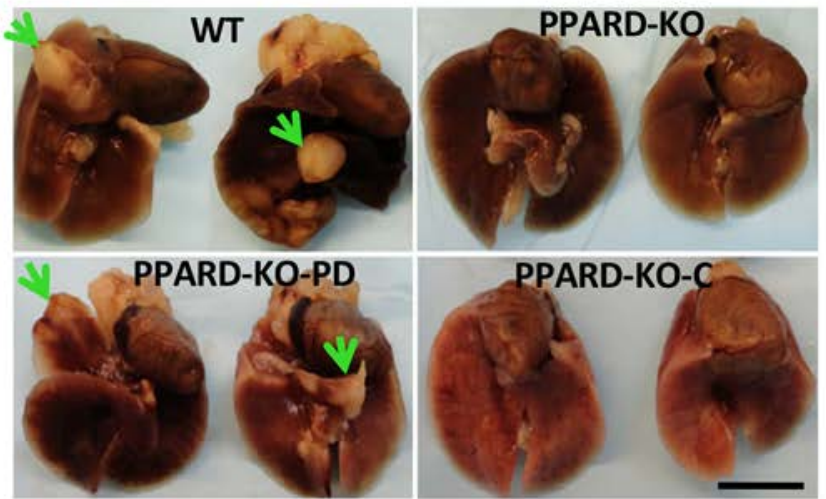

C
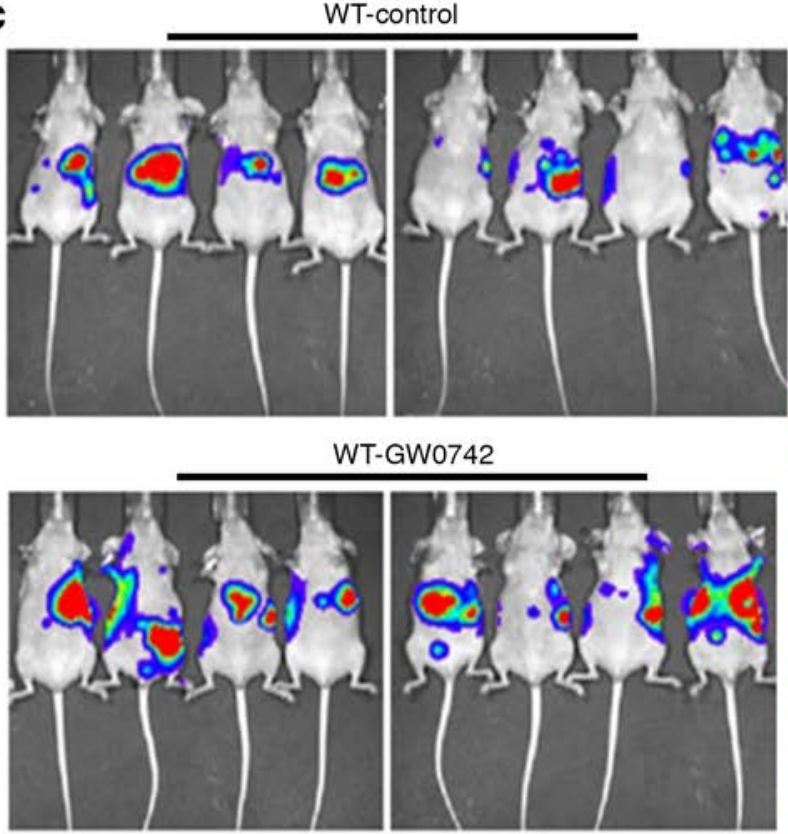

WT-GW0742

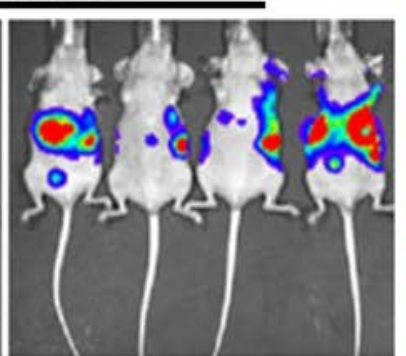

B

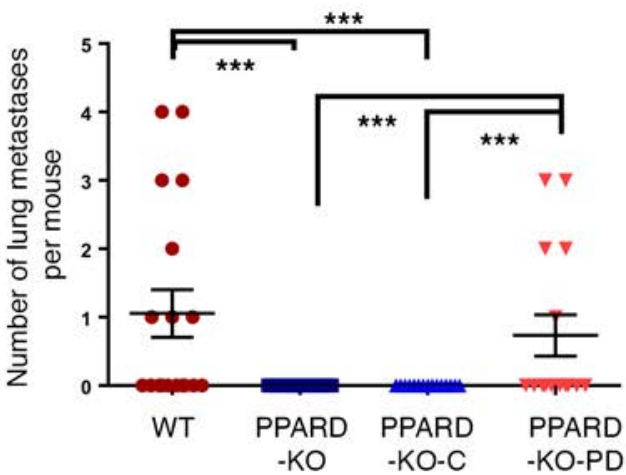

D WT-control

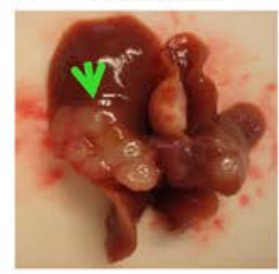

WT-GW0742

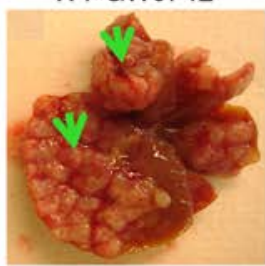

PPARD-KO-control

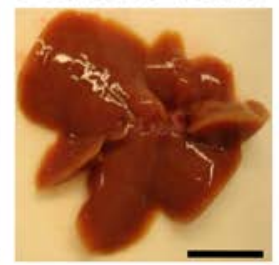

luminescence

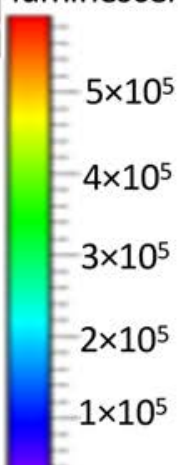

E

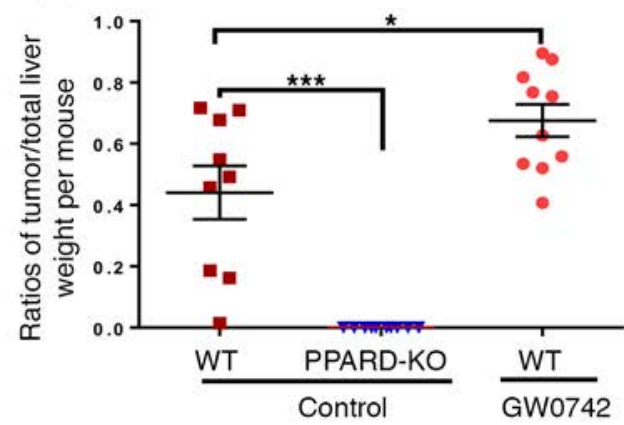

Figure 3. PPARD is critical for HCT-116 colon cancer cells' formation of lung and liver metastases in immunodeficient mice. (A and B) HCT116 parental (WT), PPARD-KO, PPARD-KO-PD, and PPARD-KO-C cells were injected via the tail vein into immunodeficient mice, and lung metastasis formation was evaluated 6 weeks later. (A) Representative photographs of formalin-fixed lungs. Arrows indicate metastases. (B) Numbers of lung metastases per mouse for each group ( $n=15-18$ per group). (C-E) HCT116 parental cells stably transfected with luciferase (WT) or HCT116-PPARD-KO cells were injected into the spleens of immunodeficient mice. The mice were then fed either a control diet or one containing $\mathrm{CW} 0742$ (1 mg/kg; $n=10-12$ per group), and liver metastasis formation was evaluated by bioluminescence imaging 2 weeks after injection. Four weeks after injection, the mice were killed, and their liver tumors were weighed and photographed. (C) Liver metastases from the indicated groups were evaluated by bioluminescence imaging 2 weeks after cell injection. (D) Representative photographs of isolated livers 4 weeks after cell injection. Arrows indicate liver metastases. (E) Ratios of liver tumor weight to total liver weight per mouse. Scale bars: $1 \mathrm{~cm}$. Values in $\mathbf{B}$ and $\mathbf{E}$ are mean $\pm \mathrm{SEM}$. Lines indicate means. ${ }^{*} P<0.01,{ }^{* * *} P<0.0001$ (ANOVA).

tasis in the same model. We injected B16-F10 melanoma cells transfected with PPARD-shRNA-A or control-shRNA into the tail veins of heterozygous PPARD-KO $\left(\mathrm{PPARD}^{+/-}\right)$mice or their WT littermates. $\mathrm{PPARD}^{+/-}$mice, generated by deleting PPARD exons 4 and 5 , which encode PPARD's binding domain, were backcrossed from their initial mixed background of B6/Sv129 to a B6 background by breeding with B6 mice for 10 generations to allow the syngeneic injection of mouse cancer cell lines of B6 background (i.e., B16-F10 cells). As reported previously, homozygous loss of PPARD function in PPARD ${ }^{-/}$mice causes high embryonic mortality rates because of PPARD's essential function in placental development (28, 29). In agreement with these prior reports, we were unable to generate $\mathrm{PPARD}^{-/-}$mice on a $\mathrm{B} 6$ background despite extensive breeding efforts and therefore used PPARD ${ }^{+/-}$mice for the experiments. Nevertheless, PPARD $^{+/-}$mice had significantly lower PPARD mRNA expression levels (Figure 5A). The decrease in PPARD mRNA expression in lung tumor foci was further augmented by PPARD downregulation in 
A

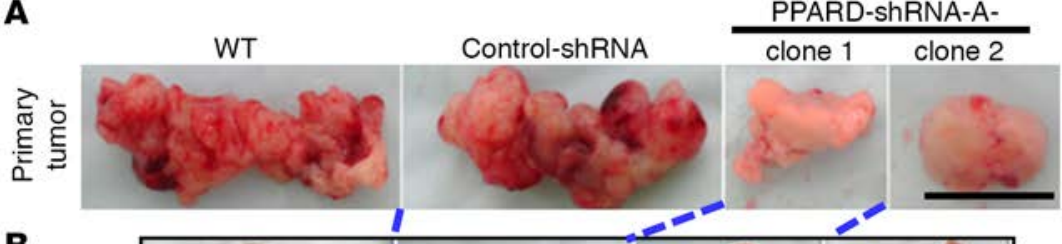

B
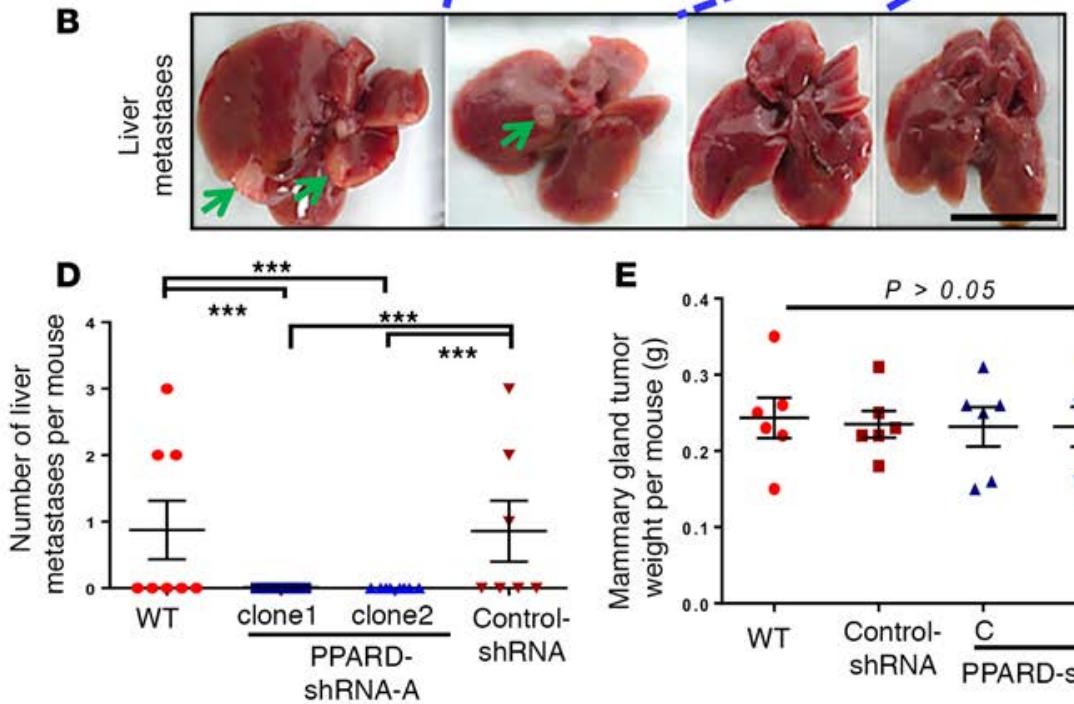

G

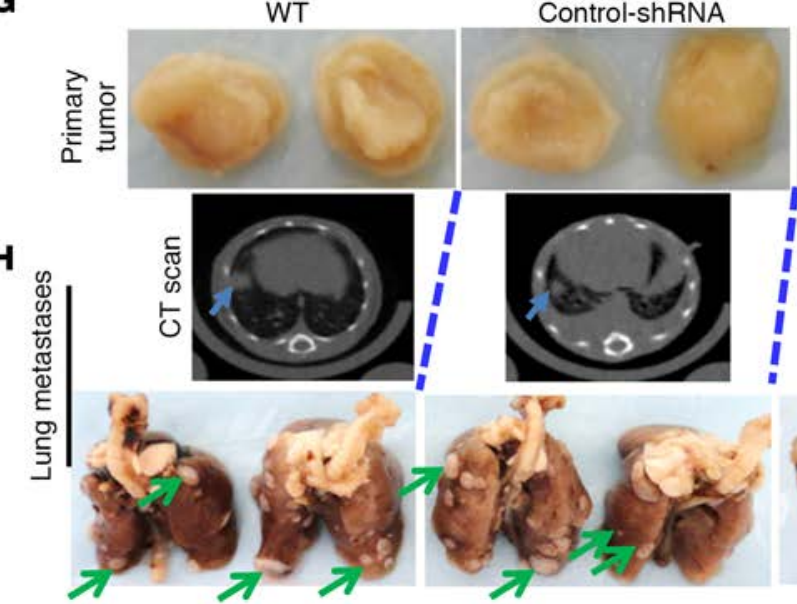

E

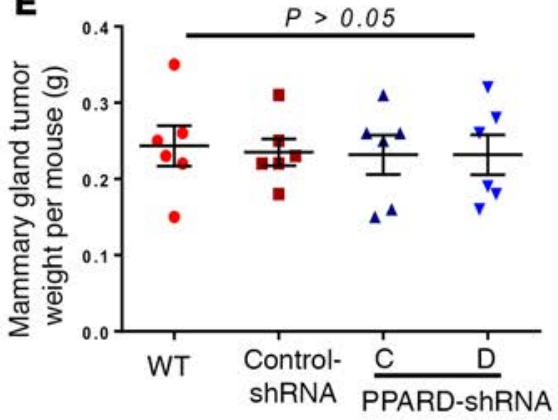

PPARD-shRNA-C
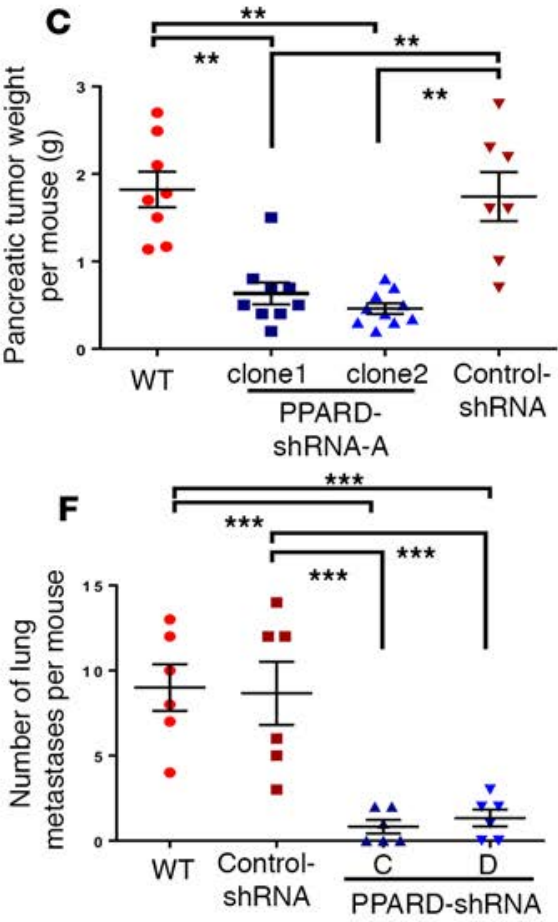

PPARD-shRNA-D
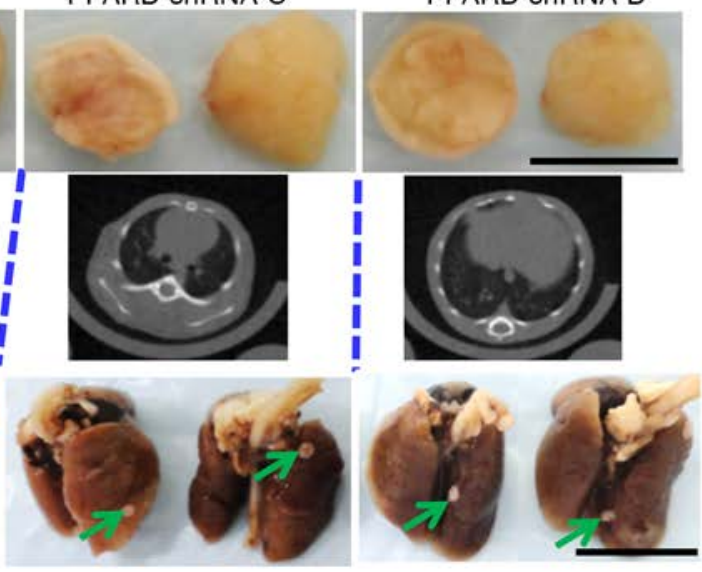

Figure 4. Effects of PPARD on the metastasis of Panc-02 and 4T1 cells in mouse orthotopic models. (A-D) WT Panc-02 cells or Panc-02 cells stably transfected with PPARD-shRNA-A (PPARD-shRNA-A-clone1 or -clone2) or control-shRNA plasmid were injected into the pancreases of syngeneic $C 57 B L / 6$ mice, and liver metastasis formation was evaluated 4 weeks later ( $n=7-10$ per group). (A and B) Representative photographs of freshly isolated pancreatic tumors (A) and livers (B). Arrows indicate liver metastases. (C) Pancreatic primary tumor weight per mouse for each group. (D) Numbers of liver metastases per mouse for each group. (E-H) WT 4T1 cells or 4T1 cells stably transduced with PPARD-shRNA-C or -D or control-shRNA lentivirus were injected into the mammary fat pads of syngeneic BALB/c mice ( $n=6$ per group), and primary mammary tumors were removed when reaching a predetermined size of 10 mm at the greatest diameter. Lung metastasis formation was evaluated 2 weeks later via lung CT and lung surface tumor count. (E) Mammary primary tumor weight per mouse for each group. (F) Numbers of lung metastases per mouse for each group. (C) Representative photographs of formalin-fixed primary mammary tumors. (H) Representative CT images of lungs (upper panel) and Davidson's solution-fixed lungs (lower panel). Arrows indicate lung metastases. All scale bars: $1 \mathrm{~cm}$. Values in $\mathbf{C}-\mathbf{F}$ are mean \pm SEM. ${ }^{* *} P<0.001$, ${ }^{* *} P<0.0001$ (2-way ANOVA for $\mathbf{C}$ and $\mathbf{E}$ and 2-sided Poisson for $\mathbf{D}$ and $\mathbf{F}$ ).

B16-F10 cells transfected with PPARD-shRNA. PPARD mRNA expression levels in lung tumor foci from PPARD-shRNA-A-clone1 or -clone2 B16-F10 cells were lower than those in lung tumor foci from control-shRNA B16-F10 cells (Figure 5B). Following the tail vein injection of control-shRNA B16-F10 cells, $\mathrm{PPARD}^{+/-}$mice developed fewer lung metastases than did WT mice (Figure 5, C and D). However, the reduction in lung metastasis formation in $\mathrm{PPARD}^{+/}$mice was much more evident following the injection of either PPARD-shRNA-A-clone1 or -clone2 B16-F10 cells (Figure 5, C and D). 
A

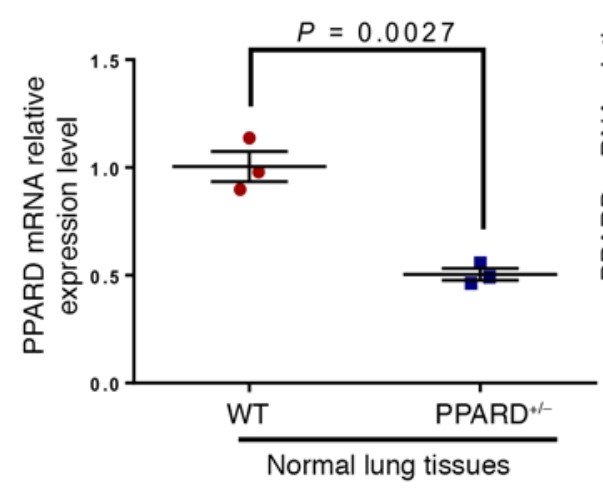

B

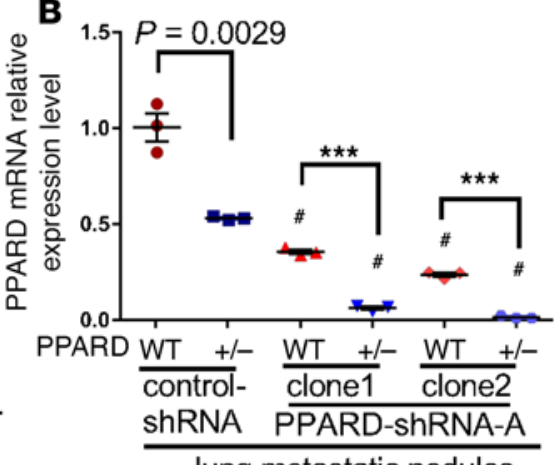

lung metastatic nodules

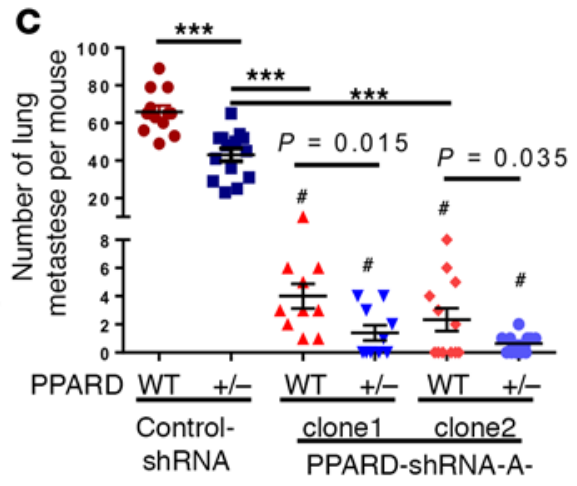

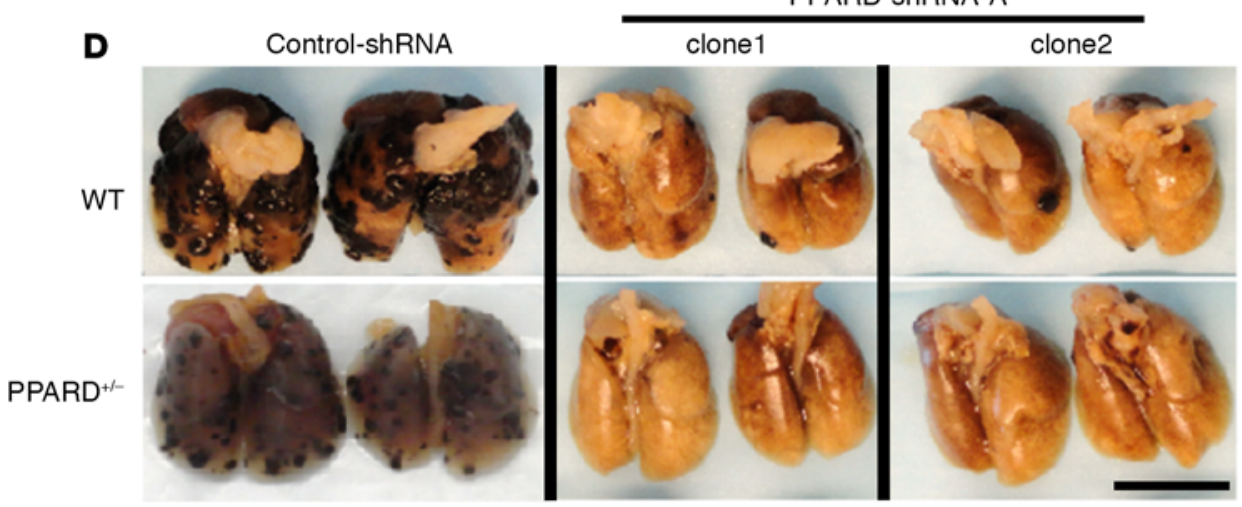

Figure 5. PPARD expression in B16-F10 cancer cells is more important for lung metastases than PPARD expression in non-cancer cells in syngeneic mice. B16-F10 melanoma cells transfected with control-shRNA or PPARD-shRNA-A (PPARD-shRNA-A-clone1 or -clone2) were injected via the tail vein into syngeneic C57BL/6 WT or heterozygous PPARD-KO mice (PPARD ${ }^{+/}$), and lung metastasis formation was evaluated 3 weeks later. (A) PPARD mRNA expression levels, as measured by qRT-PCR, in normal lung tissues from C57BL/6 WT and PPARD+/- mice. (B) PPARD mRNA expression levels, as measured by qRT-PCR, in lung metastases from the indicated groups. (C) Numbers of lung metastases per mouse for each group ( $n=10-14$ per group). (D) Representative photographs of formalin-fixed lung specimens. Scale bar: $1 \mathrm{~cm}$. Values in $\mathbf{A}-\mathbf{C}$ are mean $\pm \mathrm{SEM}$. ${ }^{* * *} P<0.0001 ;{ }^{\#} P<0.0001$ compared with control-shRNA. $P$ values were calculated with unpaired $t$ test $(\mathbf{A})$ or 2 -way ANOVA (B and $\mathbf{C}$ ).

PPARD expression in cancer cells regulates tumor angiogenesis in vivo and in vitro. Given the important role of angiogenesis in metastasis $(17,18)$, we investigated the effects that PPARD expression in cancer cells has on tumor angiogenesis. Immunohistochemical and immunofluorescence staining of CD31 to assess microvessel density revealed that PPARD downregulation in cancer cells significantly decreased angiogenesis in vivo (Figure 6, A-F, and Supplemental Figure 4A). In PPARD ${ }^{+/-}$mice, the angiogenesis inhibition induced by PPARD downregulation in non-cancer cells was further increased by PPARD downregulation in cancer cells (Figure 6, E and F, and Supplemental Figure 4).

Next, we sought to determine whether PPARD in cancer cells promoted formation of secreted proangiogenic factors to modulate angiogenesis. We screened for these factors in medium conditioned with HCT116 cells with or without PPARD overexpression. In an angiogenesis tubule formation assay using HUVECs (30), medium conditioned with HCT116 cells stably transfected with PPARD vector increased HUVEC capillary tubule formation compared with medium conditioned with HCT116 cells transfected with control vector (Figure 6, G and $\mathrm{H}$ ), thus confirming the effects of cancer cells' PPARD on angiogenesis in this model. We also confirmed that medium conditioned with HCT116 cells treated with the PPARD agonist GW0742 significantly increased HUVEC capillary tubule formation compared with medium conditioned with HCT116 cells treated with the control solvent DMSO (Figure 6, I and J). To screen for proangiogenic factors, media conditioned with PPARD vector- or control vector-transfected HCT116 cells were incubated with antibodies specific to 19 common angiogenesis-related factors immobilized on membranes. VEGF and IL-8 expression levels in the medium conditioned with PPARD vector-transfected HCT116 cells were significantly higher than those in the medium conditioned with control vector-transfected HCT116 cells (Figure 7A). These findings were confirmed by ELISA measurements of VEGF and IL-8 protein levels in cell culture media conditioned with PPARD vector- or control vector-transfected 
A

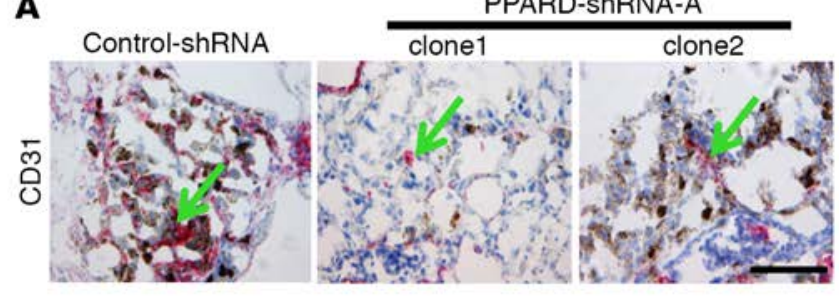

C
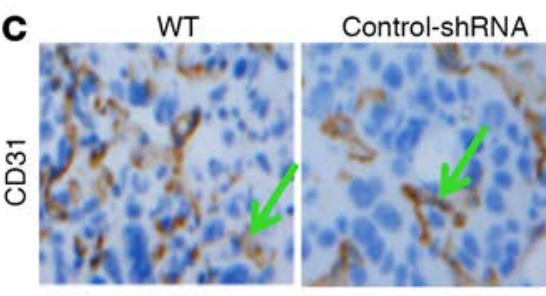

PPARD-ShRNA-B
B

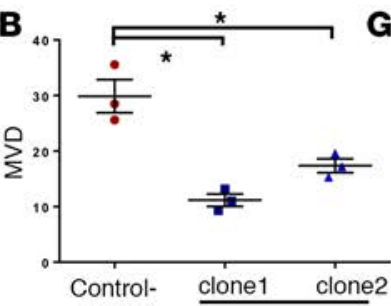

ShRNA PPARD-shRNA-A

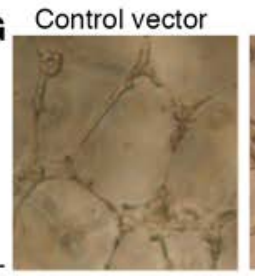

$\mathbf{H}$

\section{(1)}

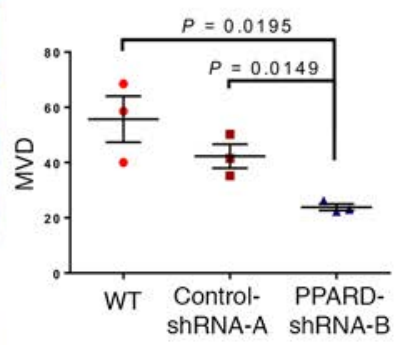

$\mathbf{F}$
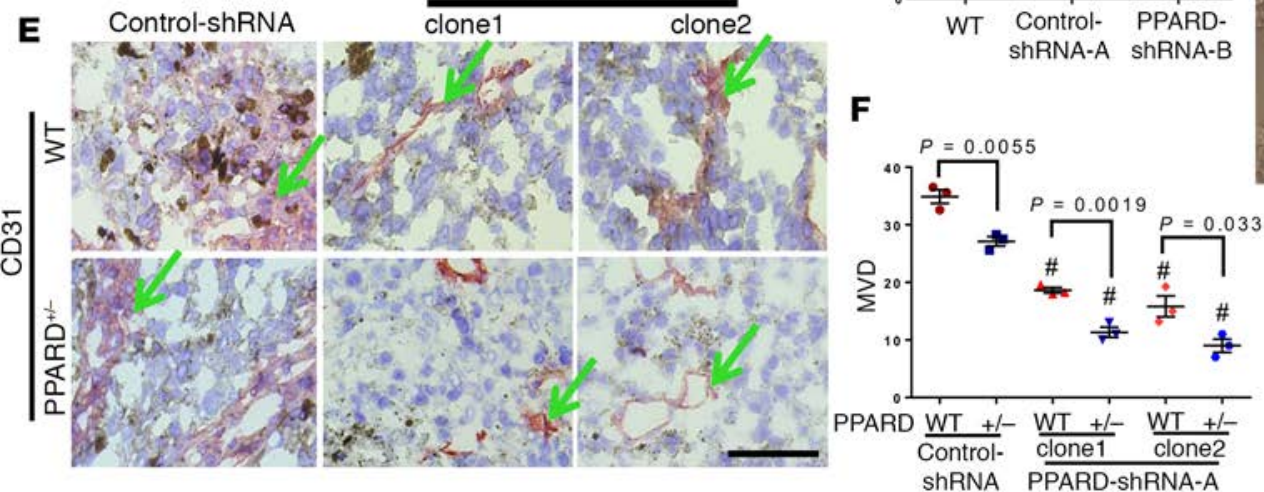
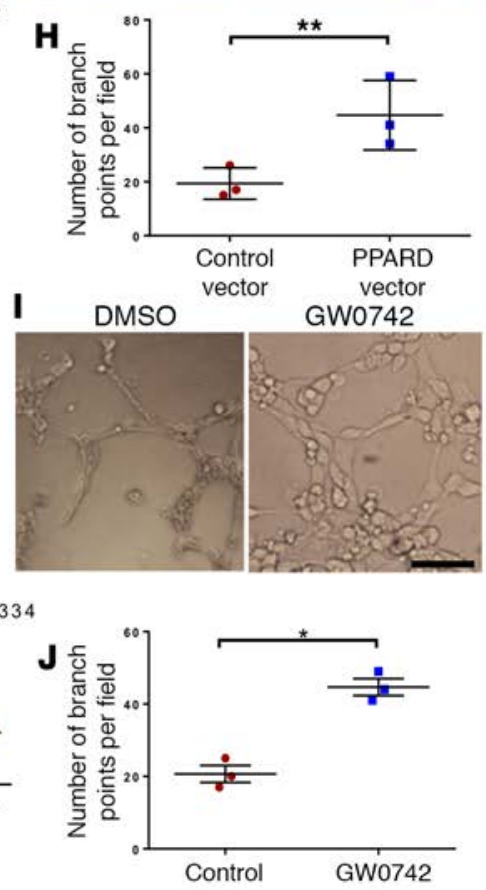

Figure 6. PPARD expression in cancer cells promotes angiogenesis in vivo and in vitro. (A-F) PPARD downregulation in cancer cells inhibits angiogenesis in vivo. (A and B) Lung metastases were formed as described in Figure 1, A and B, harvested in optimum cutting temperature compound (OCT), and assessed for the microvessel density (MVD) marker CD31 by IHC staining. (A) Representative images of IHC staining of CD31. (B) Quantification of MVD. (C and D) Lung metastases were formed as described in Figure 2, A and B, harvested in OCT, and assessed for CD31 by IHC staining. (C) Representative images of IHC staining of CD31. (D) Quantification of MVD. (E and F) Lung metastases were formed as described in Figure 5, harvested in OCT, and assessed for CD31 by IHC staining. (E) Representative images of IHC staining of CD31. (F) Quantification of MVD. Green arrows indicate tumor blood vessels. Values in $\mathbf{B}, \mathbf{D}$, and $\mathbf{F}$ are mean $\pm \mathrm{SEM}$; ${ }^{*} P<0.01$; $P<0.001$ compared with control-shRNA group. $P$ values were calculated by 1 -way (B and $\left.\mathbf{D}\right)$ or 2 -way ANOVA (F). (G and $\mathbf{H})$ PPARD overexpression in colon cancer cells promotes angiogenesis in vitro. HCT116 cells stably transfected with PPARD vector or control vector were cultured in serum-free medium for 48 hours, and the conditioned media were collected for tubule formation assay using HUVECs. (C) Representative images of tubule formation. (H) Quantification of tubule formation. (I and J) The PPARD agonist CW0742 increases tubule formation by HUVECs. HCT116 cells were treated with the PPARD agonist CW0742 $(1 \mu \mathrm{M})$ or the control solvent (DMSO) in serum-free medium for 72 hours, and the conditioned media were collected for the tubule formation assay. (I) Representative images of tubule formation. (J) Quantification of tubule formation. The values in $\mathbf{H}$ and $\mathbf{J}$ are mean \pm SEM. ${ }^{*} P<0.01 ;{ }^{* *} P<0.001$ (unpaired $t$ test). All scale bars: $100 \mu \mathrm{m}$.

HCT116 cells (Figure 7, B and C). PPARD upregulation in these cells also increased the mRNA levels of both VEGF and IL-8 (Figure 7, D and E), which suggested that PPARD transcriptionally upregulated VEGF and IL-8 expression.

Given that PPARD's regulation of VEGF had been reported previously $(11,15)$, we focused our investigation on PPARD's regulation of IL-8. Using PPARD-KO cell lines, we found that the mean IL-8 expression levels in PPARD-KO HCT116 cells were 87\% lower than those in parental WT HCT116 cells and that PPARD reconstitution in PPARD-KO cells reversed IL-8 downregulation (Figure 8A). Similarly, in vivo experiments confirmed the expression of IL-8 in lung metastases formed by HCT116 cells and PPARDKO-PD cells but not in the lung tissue of mice injected with PPARD-KO cells or PPARD-KO-C cells (Figure 8B). Compared with media conditioned with PPARD-overexpressing HCT116 cells treated with control IgG, media conditioned with PPARD-overexpressing HCT116 cells treated with an anti-IL-8 antibody had significantly less HUVEC capillary tubule formation (Figure 8, C and D), demonstrating that IL-8 has an important role in PPARD's promotion of angiogenesis. To determine whether PPARD increased 
A

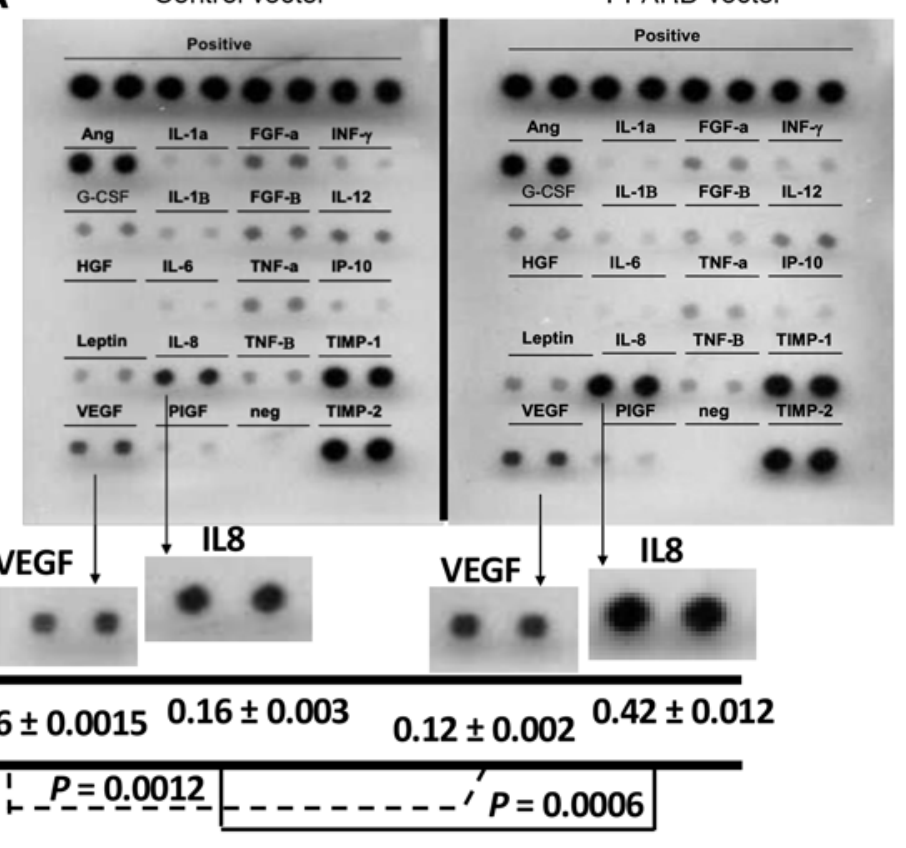

B
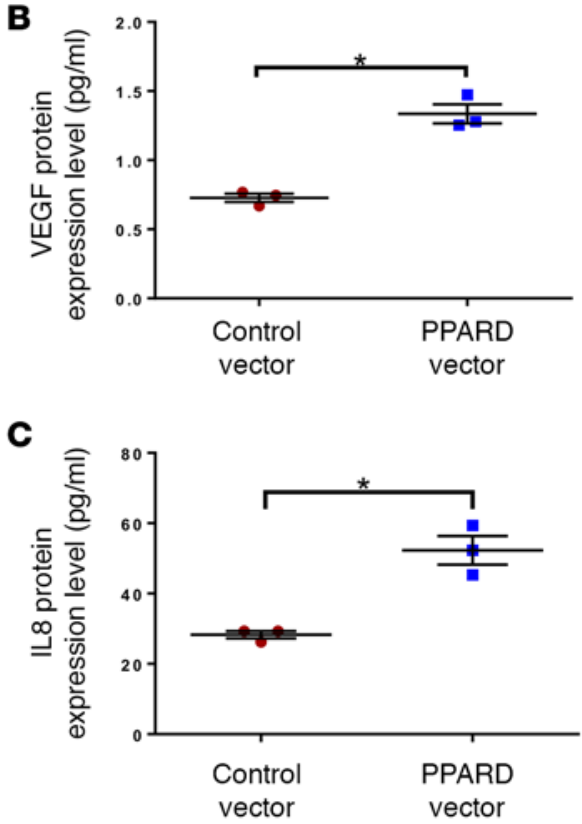

D

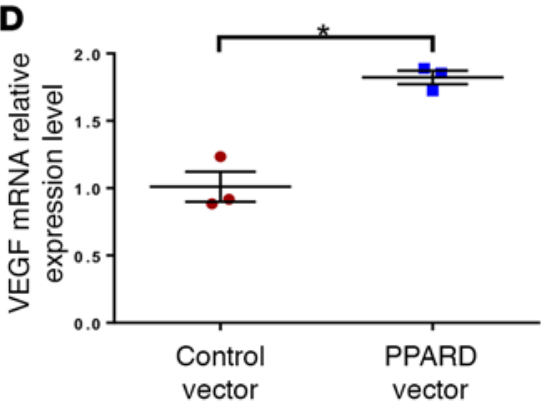

$\mathbf{E}$

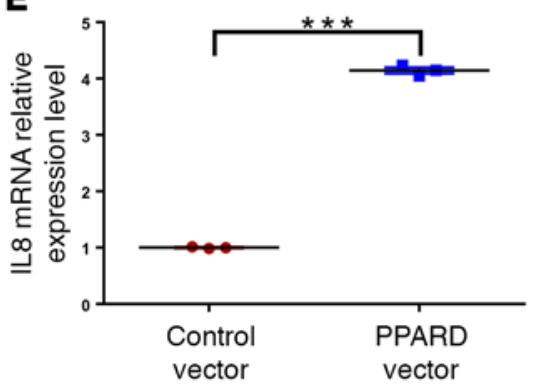

Figure 7. PPARD overexpression in cancer cells promotes their secretion of proangiogenic factors. (A) The media conditioned with HCT116 cells stably transfected with PPARD vector or control vector were collected for an angiogenesis antibody array assay. VEGF and IL-8 protein expression levels are shown. Band densities are presented as the ratios of the positive dots. (B-E) VEGF and IL-8 protein levels in the conditioned media collected as described in $\mathbf{A}$ were measured by ELISA (B and $\mathbf{C}$ ), and VEGF and IL-8 mRNA expression levels in the cells were measured by qRT-PCR ( $\mathbf{D}$ and $\mathbf{E})$. Values are mean \pm SD. ${ }^{*} P<0.01,{ }^{* *} P<0.0001$ (unpaired $t$ test).

IL-8 via direct transcriptional regulation, we transfected HCT-116 and PPARD-KO cells with IL-8 luciferase reporter promoter deletion constructs. IL-8 promoter activity, except for that of the -133 - to +50 -bp construct, was reduced in PPARD-KO cells compared with WT cells (Figure 8E). In confirmatory experiments with different cell lines and complementary PPARD expression modulation in LoVo cells, PPARD overexpression by PPARD vector transfection increased the IL-8 promoter activity of constructs more than 133 bp upstream of the initiation codon (Supplemental Figure 5A). ChIP-quantitative PCR (ChIP-qPCR) assays revealed that PPARD did bind to the IL-8 promoter region between -276 and -133 bp (Figure $8 \mathrm{~F}$ ) as predicted by earlier promoter deletion experiments (Figure $8 \mathrm{E}$ and Supplemental Figure 5A).

To determine the clinical relevance of our findings, we measured PPARD and IL- 8 expression levels in paired samples of normal colonic mucosa and cancerous mucosa from 22 patients with stage III colon cancer treated at the University of Texas MD Anderson Cancer Center. The clinical characteristics of the patients are summarized in Supplemental Table 1. In 21 of the 22 patients, the RNA integrity numbers for the normal and cancerous mucosa samples were above 5 , the minimal acceptable level for comparable analyses (31). The exclusion of 1 patient's data did not substantially alter the results (Supplemental Figure 6, A-D). PPARD levels were significantly higher in cancerous mucosa (median: 6.11 ; interquartile range [IQR]: 2.7-10.33) than in normal mucosa (median: 1.17; IQR: 0.92-2.02) (Figure 9A). IL-8 levels were 
A

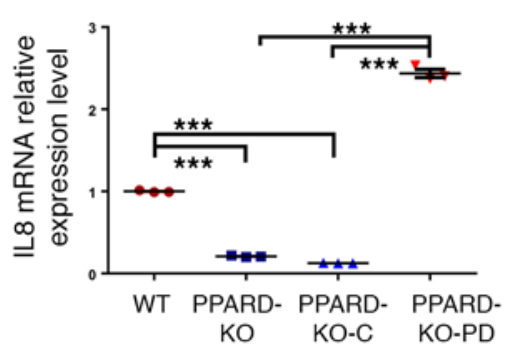

B

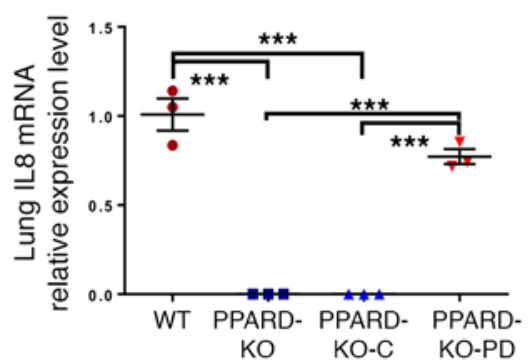

C

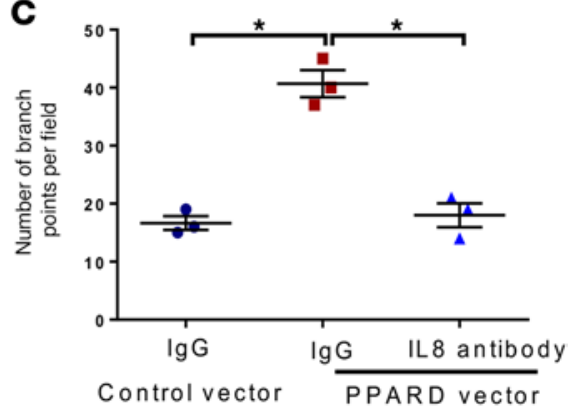

D
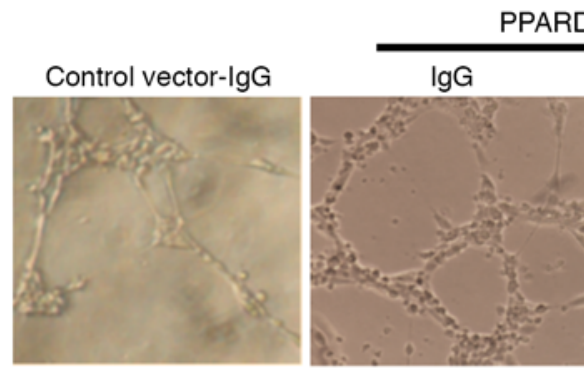

PPARD vector

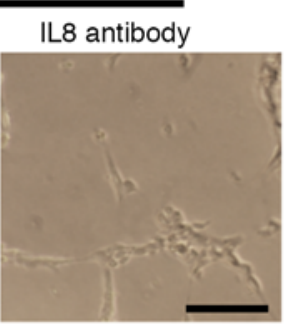

$\mathbf{F}$

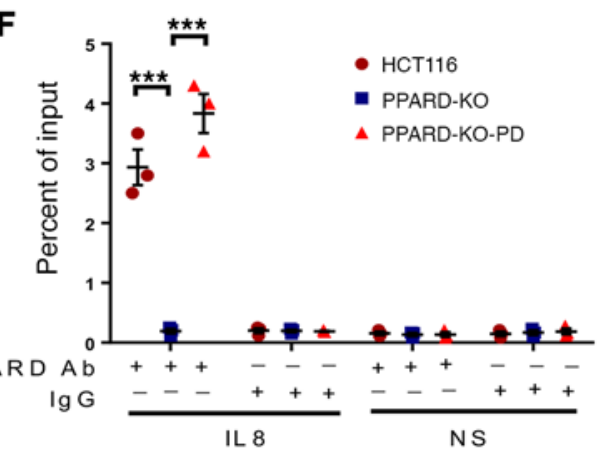

E

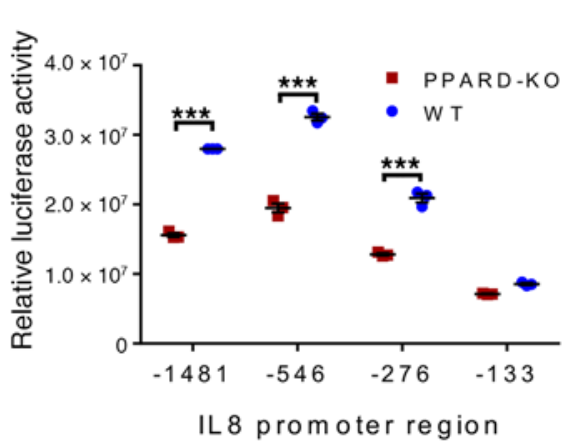

Figure 8. PPARD in cancer cells positively regulates IL-8 expression. (A and B) IL-8 mRNA levels were measured in HCT116-WT, PPARD-KO, PPARD-KO-PD, and PPARD-KO-C cells (A) and in xenograft lung metastases from mice injected with HCT116 parental (WT) or PPARD-KO-PD cells and in the lung tissues from mice injected with HCT116 PPARD-KO or PPARD-KO-C cells as described in Figure 3, A and B (B) by qRT-PCR. (C and D) Anti-IL-8 antibody inhibits tubule formation of HUVECs. HCT116 cells stably transfected with PPARD vector or control vector were treated with either nonspecific IgC or an anti-IL-8 neutralized antibody for 72 hours, and the conditioned media were collected for a tubule formation assay. (C) Quantification of tubule formation. (D) Representative images of tubule formation. (E) HCT116 WT and PPARD$\mathrm{KO}$ cells were transiently transfected with the PCL4.16 luciferase reporter vector containing the indicated IL- 8 promoter regions and the PSV- $\beta$-galactosidase vector. Luciferase activity was measured 24 hours later and normalized for $\beta$-galactosidase activity. (F) PPARD binds to the IL-8 promoter to increase IL-8 expression. A 113-bp fragment of the human IL-8 promoter was amplified by $\mathrm{QPCR}$, and the percent of input was used to measure the ability of PPARD to bind to the IL-8 promoter. Scale bar: $100 \mu \mathrm{m}$. Values in A-C, $\mathbf{E}$ and $\mathbf{F}$ are mean $\pm \mathrm{SEM}$. ${ }^{*} P<0.01$, ${ }^{* * *} P<0.0001$ (2-way [A and $\mathbf{B}$ ] or 1-way [C and $\mathbf{F}$ ] ANOVA; unpaired $t$ test [E]).

also significantly higher in cancerous mucosa (median: 82.5 ; IQR: 14.47-383.8) than in normal mucosa (median: 0.76; IQR: 0.2-3.63) (Figure 9B). PPARD mRNA expression levels were significantly correlated with IL-8 mRNA levels in cancerous mucosa (Figure 9C).

Cellular mechanisms of cancer cell PPARD's promotion of metastasis. To further gain insight into the mechanisms by which PPARD expression in cancer cells promotes metastasis, we performed a transcriptome-wide analysis using RNA sequencing (RNA-Seq) to compare parental WT and PPARD-KO HCT116 cells, whose abilities to form metastases in vivo differ profoundly (Figure 3, A and B). Using cluster analyses with a cut-off false discovery rate of 0.001 and a fold change greater than 2, we identified 416 genes that were significantly differentially expressed between WT and PPARD-KO HCT116 cells (Figure 10, A and B). The gene expression patterns were significantly different by PPARD genetic KO status in HCT116 cells but consistent within the groups with the same PPARD expression status (Figure 10A). Among the differentially expressed genes, 311 were downregulated and 105 were upregulated in the PPARD-KO cells compared with WT cells (Figure 10B).

To better understand the way in which the differences in gene expression by PPARD status are related to metastasis, we used Ingenuity Pathway Analysis to assess the functions of the differentially expressed genes. The top 4 biological functions identified for the differentially expressed genes were "cancer," "cellular growth and proliferation," and "cellular movement"; "cell death and survival" was also among the top 
A

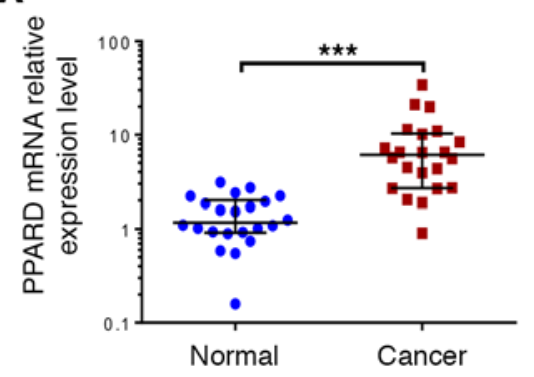

B

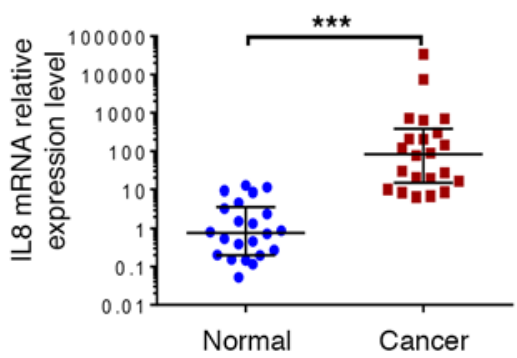

C

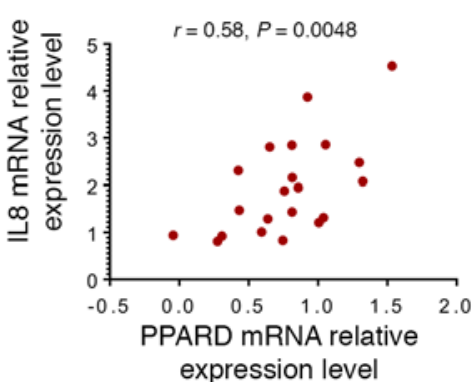

Figure 9. Expression and correlation of PPARD and IL-8 in human colorectal cancer. (A-C) Scatter plots of PPARD (A) and IL-8 (B) mRNA expression levels in paired normal and cancerous mucosa samples of colorectal cancer patients $(n=22)$. Values are median with interquartile range. ${ }^{* * *} P<0.0001$ (paired $t$ test). (C) Correlation between IL-8 and PPARD mRNA expression levels in samples described in $\mathbf{A}$ and $\mathbf{B}$. Values are the log-transformed mean measurements per patient for each variable. Statistical analysis was performed with Spearman's correlation test.

biological functions identified (Figure 10C). These functions suggest that PPARD expression significantly influences important metastasis mechanisms such as cell invasion and migration.

We next sought to determine the clinical relevance of the differentially expressed genes in the transcriptome. Genes with more than 3-fold higher expression in WT HCT116 cells than in PPARD-KO HCT116 cells were screened for their association with PPARD mRNA expression in the provisional TCGA (The Cancer Genome Atlas) colorectal adenocarcinoma database (382 cases) in cBioPortal (http://www.cBioPortal.org) (32, 33). Twenty-three of the differentially expressed genes had significant association (i.e., a tendency toward co-occurrence) with PPARD expression $(P<0.05$; log odds ratio $>1.5)$ in the TCGA colorectal adenocarcinoma database (Supplemental Table 2). Of these 23 genes, 7 were linked to metastasis by PubMed literature searches: gap junction protein alpha 1 (GJA1), vimentin (VIM), secreted protein, acidic, rich in cysteine (SPARC), neuregulin-1 (NRG1), CXCL8 (IL-8), stanniocalcin-1 (STC1), and synuclein gamma (breast cancer-specific protein 1) (SNCG). The IL-8 findings validated our earlier findings regarding IL-8; the NRG1 findings are consistent with our previously reported results that PPARD upregulated NRG1 in a mouse model of intestinal PPARD overexpression (34).

Quantitative real-time PCR (qRT-PCR) analyses confirmed that PPARD KO significantly reduced SNCG, STC1, SPARC, and GJA1 expression in PPARD-KO HCT116 cells (Figure 10, D-G) and in different other cancer cell lines (e.g., SW620, Hey8A) with siRNA-induced PPARD knockdown (Supplemental Figure 7, A-H). ChIP-qPCR assays confirmed that PPARD binds to the region of the GJA1 promoter that has PPAR binding sites (Figure 10H).

VIM expression in parental WT HCT116 cells was more than 32-fold higher than that in PPARDKO cells (Supplemental Table 2). To further assess the clinical relevance of the link between PPARD and vimentin, we interrogated the provisional TCGA colorectal, breast cancer, lung, and prostate cancer databases (352-1,065 patients/database) in cBioPortal. PPARD and VIM mRNA expression levels were significantly correlated in the clinical samples of colorectal, breast, lung, and prostate cancers (Figure 11, A-D).

Because VIM is a strong marker of epithelial-mesenchymal transition (EMT), we investigated the effects of PPARD on EMT. Of the genes we found to be significantly differentially expressed between WT and PPARD-KO HCT116 cells, 11 are strongly linked to EMT (e.g., E-cadherin, ZEB1) (Figure 11E). In independent experiments, PPARD KO in HCT-116 upregulated E-cadherin and GRHL2 and downregulated VIM, SNAIL, TWIST, and ZEB1 expression levels; reexpression of PPARD reversed these effects (Figure 11, F-H). In independent experiments, PPARD downregulation by siRNA upregulated E-cadherin and downregulated VIM in SW480, SW620, and LoVo colon cancer cells (Supplemental Figure 8, A-F). Furthermore, genetic deletion of PPARD altered HCT116 cell morphology from mesenchymal to epithelial; reexpression of PPARD reversed this effect (Figure 11I).

Given the mechanistic contribution of EMT to invasion and migration, we next assessed the effects of PPARD on cell invasion and migration. Compared with WT HCT116 cells, PPARD-KO HCT116 cells had significantly reduced migration and invasion; reexpression of PPARD reversed these effects (Figure 11, J-L). Similarly, PPARD downregulation by shRNA in B16-F10 cells significantly reduced their invasion, migration, and clonogenic growth in anchorage-independent conditions (Supplemental Figure 9, A-E) 


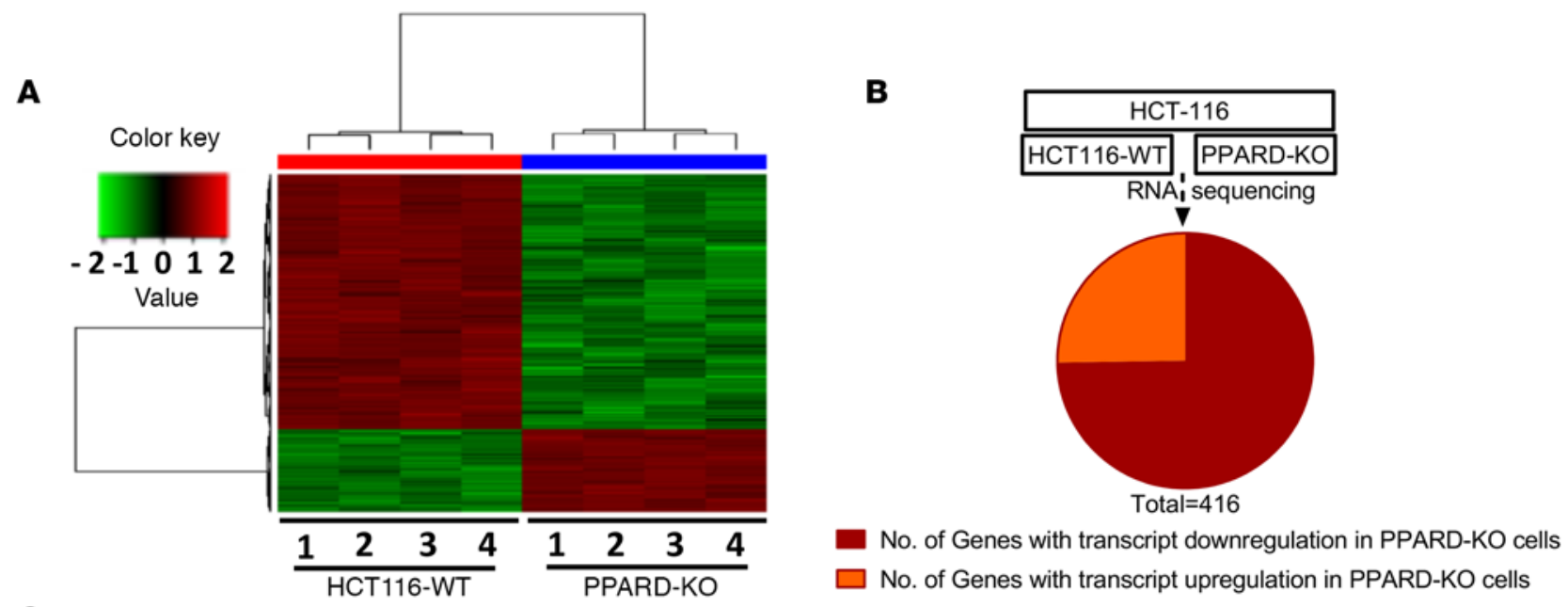

C Top 10 IPA functions accociated with the DEGs between HCT116-WT and PPARD-KO
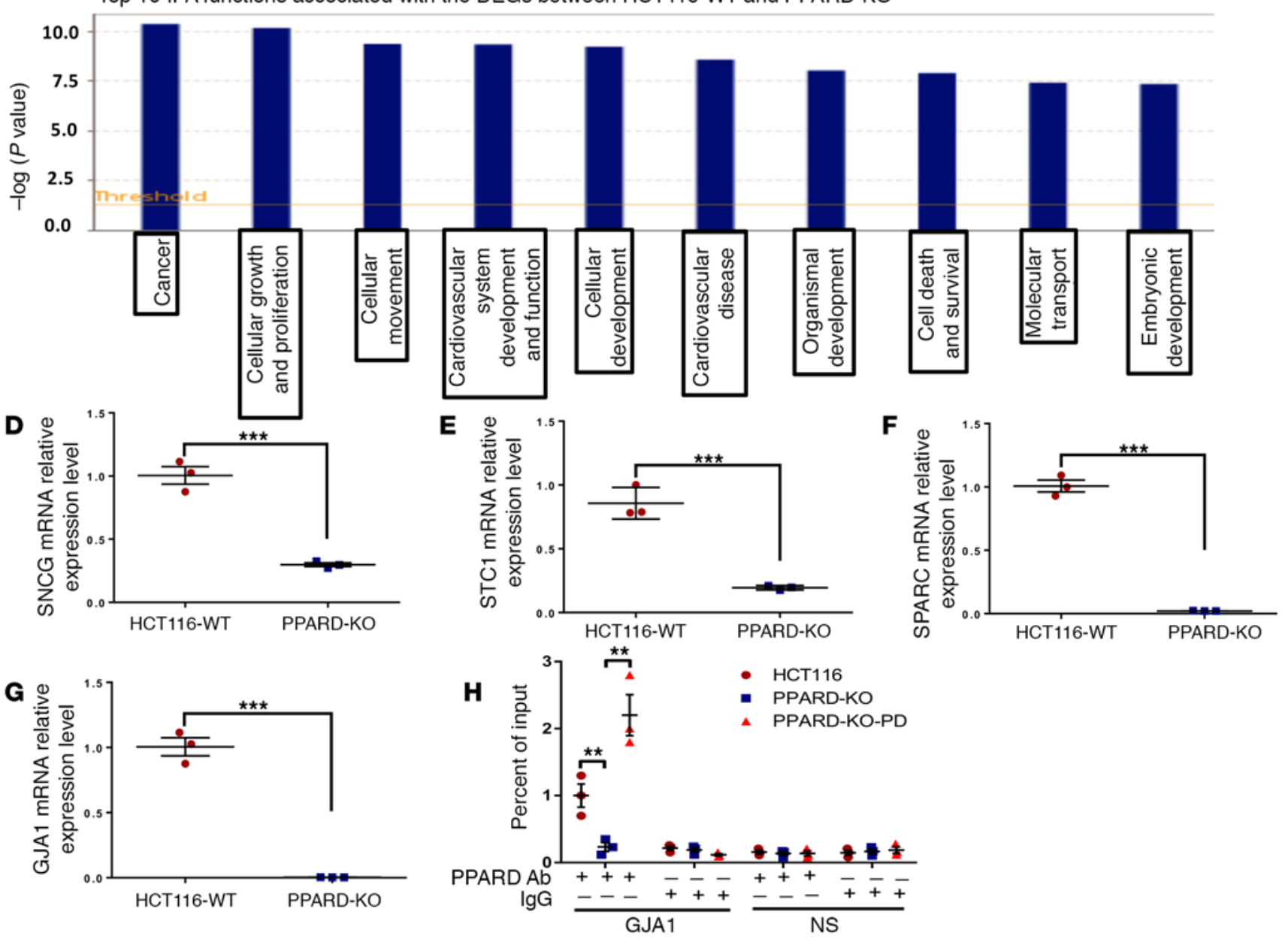

Figure 10. Transcriptome profiles of HCT116 cells with or without PPARD genetic deletion. (A and B) Heat map of 416 transcripts that were differentially expressed between HCT116 parental cells (WT) and PPARD-KO cells using a cut-off false discovery rate of 0.001 and a fold change of $>2$. Among the differentially expressed genes (DEGs), 311 were downregulated and 105 were upregulated in PPARD-KO cells compared with WT cells. (C) Canonical pathway analysis of genes differentially modulated by PPARD. The DEGs shown in $\mathbf{A}$ and $\mathbf{B}$ were subjected to Ingenuity Pathway Analysis, which identified the top 10 functions associated with the genes. $P$ values represent the probability that random chance could produce the association between the identified canonical pathways and PPARD expression. (D-G) Validation of RNA-Seq results by qRT-PCR analysis for 4 metastasis-related genes upregulated by PPARD (SNCG, STC1, SPARC, and GJA1). (H) PPARD binding to the GJA1 promoter. A 107-bp fragment of the human GJA1 promoter was amplified by $q P C R$, and the percent of input was used to measure the ability of PPARD binding to the GJA1 promoter. Values in $\mathbf{D}-\mathbf{H}$ are mean \pm SEM. ${ }^{* *} P<0.001 ;{ }^{* *} P<0.0001$ (unpaired $t$ tests for $\mathbf{D}-\mathbf{C}$ and 1-way ANOVA for $\mathbf{H}$ ). 
A
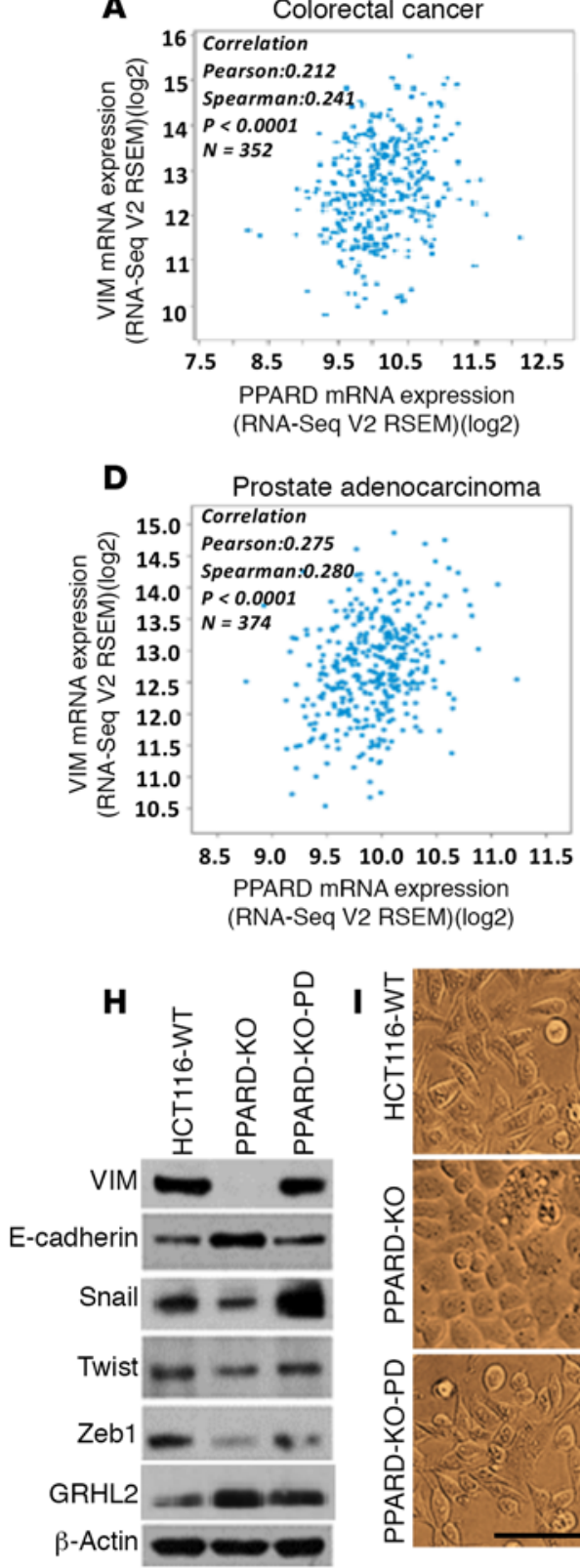

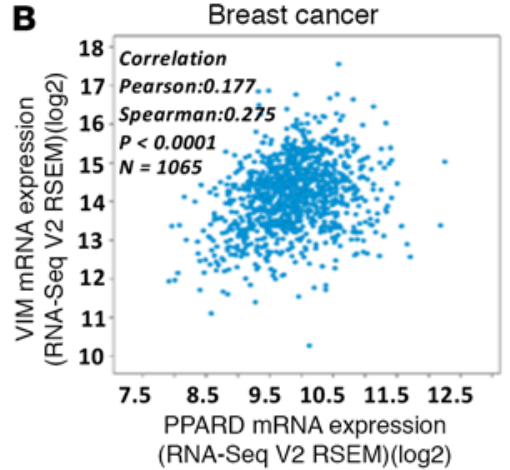

$\mathbf{E}$

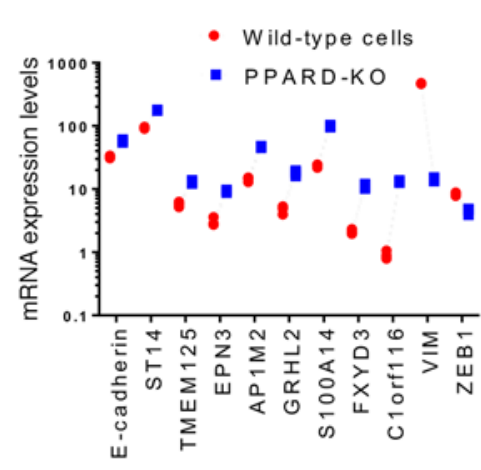

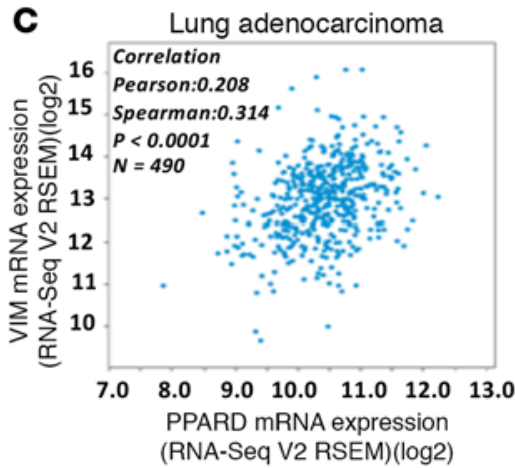

$\mathbf{F}$

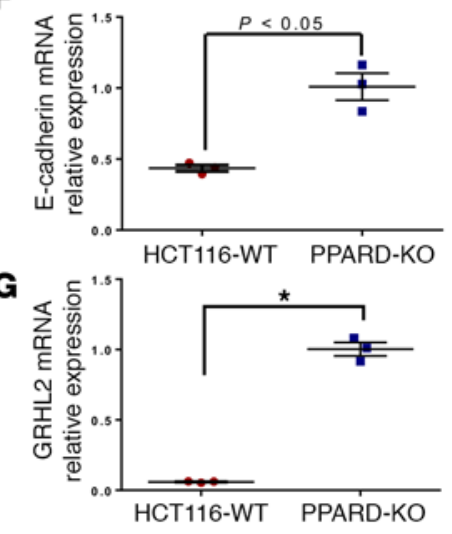

J

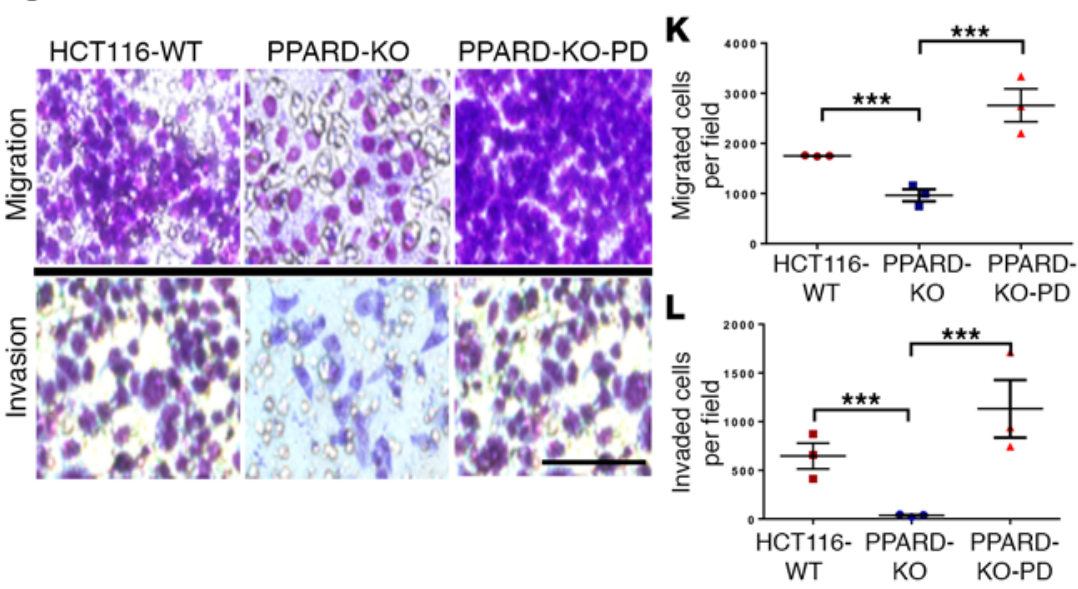

Figure 11. PPARD expression in cancer cells promotes EMT and cancer cell migration and invasion. (A-D) Relationship between vimentin (VIM) and PPARD in colorectal (A), breast (B), lung (C), and prostate (D) cancers in large TCGA database cohorts. (E) EMT-related genes that RNA-Seq analysis revealed to be differentially expressed between HCT116 WT and PPARD-KO cells. (F and $\mathbf{G}$ ) Validation of the RNA-Seq results shown in E by qRT-PCR analysis for E-cadherin (F) and GRHL2 (G). (H) PPARD increases the expression levels of EMT-related genes as measured by Western blotting. (I) Representative photographs of monolayer-cultured HCT116 WT, PPARD-KO, and PPARD-KO-PD cells. (J-L) PPARD's effects on the migration and invasion of HCT116 parental (WT), PPARD-KO, and PPARD-KO-PD cells. (J) Representative photomicrographs of cell migration (top row) or invasion (bottom row). (K and L) Migrated cells $(\mathbf{K})$ and invaded cells $(\mathbf{L})$ in at least 4 random individual fields per insert membrane were counted. Scale bars: $100 \mu \mathrm{m}$. Values are mean \pm SEM. ${ }^{*} P<0.01 ;{ }^{* * *} P<0.0001$ (unpaired $t$ test for $\mathbf{F}$ and $\mathbf{G}$ and 1-way ANOVA for $\mathbf{K}$ and $\mathbf{L}$ ).

PPARD upregulation and metastasis risk in humans. Our preclinical findings indicated that PPARD is important for metastasis formation and is upregulated in stage III colon cancer. Therefore, we assessed the association of PPARD upregulation with metastasis risk in colon cancer patients. Among patients with stage III colon cancer, the PPARD mRNA levels in cancerous mucosa from patients who developed metastases after surgical resection (median: 8.67; IQR: 6.299-20.15) were higher than those from patients who did not develop metastases (median: 3.32; IQR: 2.191-5.998) (Figure 12A). In a larger cohort of colorectal cancer 
patients ( $n=152$; Supplemental Table 3), immunohistochemical staining for PPARD expression in cancer cells revealed that PPARD was upregulated in tumors in all cases for which paired normal and cancer tissue samples were available (Figure 12, B-D). The composite expression scores for nucleic and cytoplasmic staining in normal tissue (median: 1.00; IQR: 1.00-2.25) were lower than those in paired tumors (median: 14.00; IQR: 11.88-16.00) (Figure 12B). Furthermore, in tumor tissues, PPARD was expressed in both the cytoplasm (median: 8; IQR: 6-10) (Figure 12C) and nucleus (median: 6.5; IQR: 4.5-9.0), whereas in normal tissues, PPARD was expressed in the nucleus only (median: 1.00; IQR: 1.00-2.25) (Figure 12C). In this cohort, patients with stage III colon cancer with high PPARD expression had significantly reduced distant metastasis-free survival (hazard ratio [HR]: 5.61; 95\% CI: 1.38-13.36; $P=0.012$, log-rank test) (Figure 12E).

To further assess the effects of PPARD on distant metastasis-free survival, we used the PrognoScan database (http://www.prognoscan.org), which provides a tool that utilizes information from a large collection of publicly available cancer microarray data sets with clinical annotation to evaluate the relationship between prognosis and the expression of specific genes (35). We selected studies that reported on disease-specific survival, distant metastasis-free survival, distant recurrence-free survival, and relapse-free survival and that had significant $P$ values (corrected and Cox $P$ values). All 7 identified studies showed that high PPARD expression was associated with poor prognosis (Supplemental Table 4). The studies of distant metastasis-free survival and distant recurrence-free survival were relatively large $(n=140-286)$ and reported HRs of 1.98 to 2.69 (Figure 12, F-H, Supplemental Table 4). Similarly, studies of relapse-free survival or disease-specific survival consistently showed that patients with breast or lung cancer with high PPARD expression had worse prognosis (HRs of 2.02-2.93) (Supplemental Figure 10, A-C; Supplemental Table 4). Higher PPARD expression was also associated with poorer prognosis in other publically available databases: In the Prediction of Clinical Outcomes from Genomic Profiles (PRECOG) database (http://precog.stanford.edu), the global unweighted survival $Z$ scores for PPARD effects in all included human cancer studies was $2.6(P<0.05)$. This global unbiased meta-analysis indicator confirms the negative impact PPARD has on the survival of patients with various cancers. Furthermore, in several large studies $(>200$ patients/study) included in the PRECOG database portal, high PPARD expression had a significantly negative association with poor prognosis in patients with various cancers (Supplemental Figure 11, A, C, and E). Higher PPARD expression was associated with a significant reduction in distant metastasis-free survival in a cohort of 1,609 breast cancer patients for whom distant metastases survival data were publically available via the KMplot database portal (http://www.kmplot.com) (36) (Supplemental Figure 11G). High IL-8 expression was similarly associated with poor prognosis in these studies (Supplemental Figure $11, \mathrm{~B}, \mathrm{D}, \mathrm{F}$, and $\mathrm{H})$.

\section{Discussion}

Our current results show that PPARD expression in cancer cells critically impacts metastasis development in various preclinical models in vivo, regulates several essential pro-metastatic mechanisms (angiogenesis, EMT, and cancer cell invasion and migration), and strongly affects the metastasis-free survival of patients with various cancers. These results demonstrate that PPARD expression in cancer cells strongly impacts their metastatic potential.

We found that specific PPARD modulation in cancer cells profoundly influenced metastasis development in commonly used metastatic preclinical models in vivo. In tail vein metastasis assays, PPARD downregulation in mouse B16-F10 melanoma or LLC lung cancer cells significantly inhibited metastasis. In a spontaneous metastasis model of orthotopically injected mouse Panc-02 pancreatic cancer or 4T1 breast cancer cells, PPARD downregulation blocked the development of hepatic metastasis. In addition, genetic deletion of PPARD in human HCT-116 colon cancer cells blocked their ability to form lung metastasis in tail vein assays, and this ability was restored when PPARD was reconstituted. Furthermore, splenic injections of HCT-116 cells with genetic deletion of PPARD did not give rise to hepatic metastases, whereas a PPARD agonist enhanced parental WT HCT-116 cells' formation of hepatic metastases. These findings strongly argue against the proposed antitumorigenic role of PPARD in cancer cells and demonstrate the necessity of PPARD in cancer cells for metastasis. Furthermore, our data demonstrate that PPARD downregulation in cancer cells inhibited metastasis more profoundly than did PPARD downregulation in non-cancer cells, which establishes the specific role of PPARD in cancer cells in promoting metastases and challenges the previous proposition that PPARD expression in cancer cells versus non-cancer cells has bidirectional and opposing effects on tumorigenesis $(7,19)$. 
A

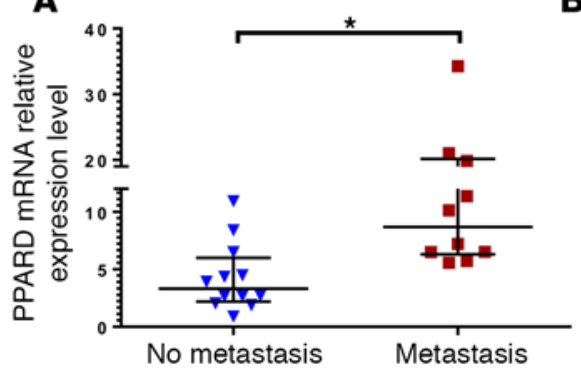

D
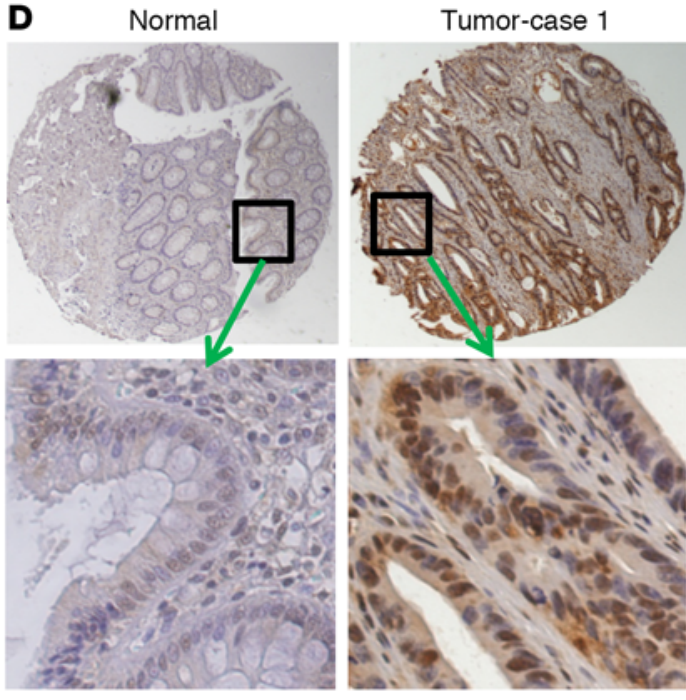

G

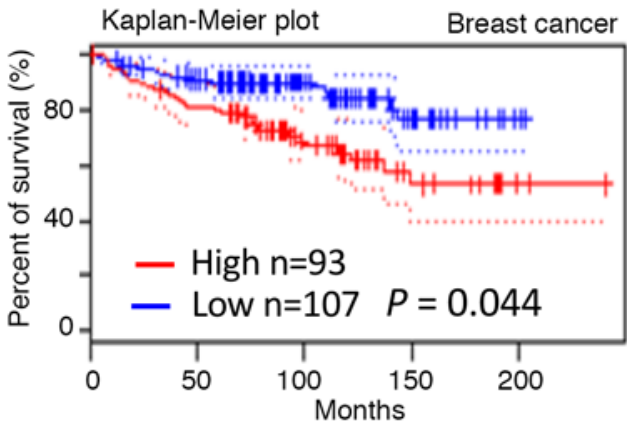

B

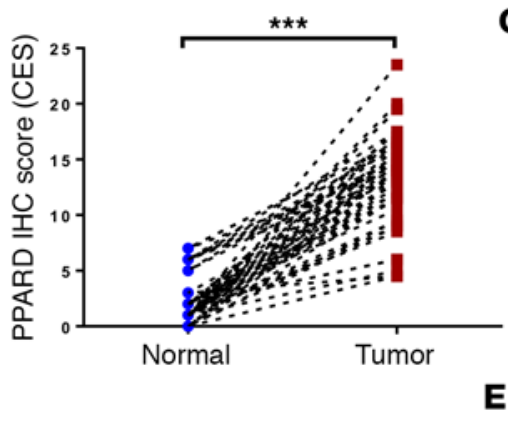

C

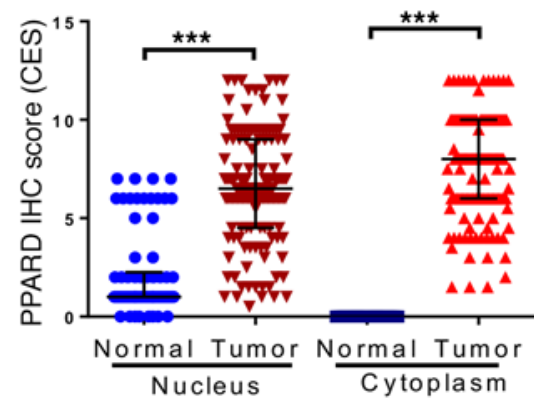

E Kaplan-Meier plot colorectal cancer

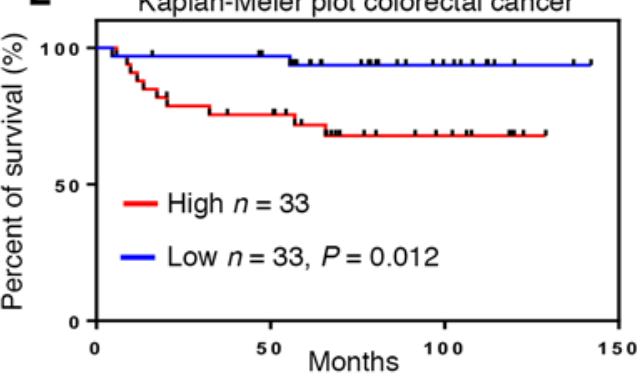

Tumor-case 2

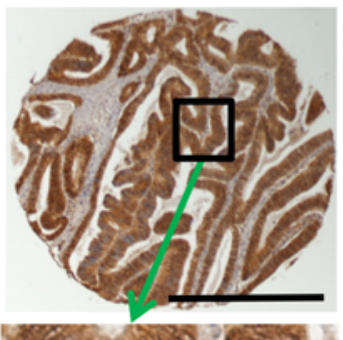

F Kaplan-Meier plot

Breast cancer
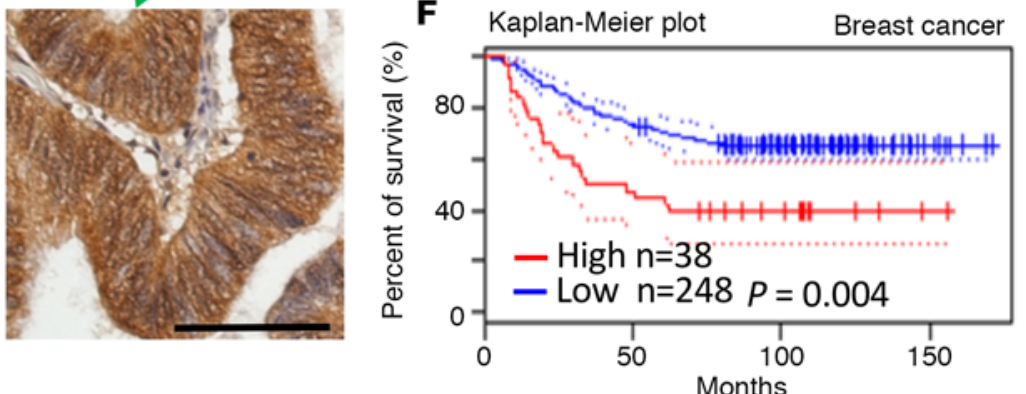

H

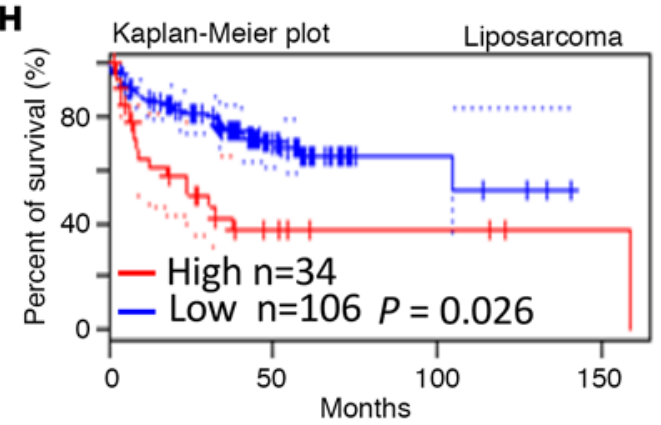

Figure 12. PPARD is upregulated in human colorectal cancer and negatively correlated with cancer patient survival. (A) The PPARD mRNA expression levels in cancerous mucosa samples from colon cancer patients who developed metastasis $(n=10)$ are significantly higher than those from the patients who did not develop metastasis $(n=12)$. (B-D) PPARD protein expression, as measured by IHC staining, in 152 colon cancer patients treated at the MD Anderson Cancer Center between 2001 and 2009. (B) Total composite expression scores (CESs) for IHC staining of PPARD in the nuclei and cytoplasm of normal and paired colorectal tumor tissues $(n=66)$. (C) Separated CESs for IHC staining of PPARD in the nuclei and cytoplasm of normal and paired colorectal tumor tissues ( $n=66$ for normal and $n=152$ for tumor samples). Values for $\mathbf{A}-\mathbf{C}$ are medians with interquartile ranges. ${ }^{*} P<0.01 ;{ }^{* *} P<0.0001$ (unpaired $[\mathbf{A}]$ or paired $[\mathbf{B}$ and $\mathbf{C}] t$ test). (D) Representative photographs of IHC staining of PPARD in normal and tumor colorectal tissues. Scale bars: upper $(1 \mathrm{~mm})$; lower $(100 \mu \mathrm{m})$. (E) Kaplan-Meier estimates of distant metastasis-free survival as a function of high versus low PPARD expression in tumors from patients with stage III colorectal cancer in the cohort described in B-D. (F-H) Kaplan-Meier estimates of distant metastasis-free survival as a function of high versus low PPARD expression in tumors from 2 cohorts of breast cancer patients (F and $\mathbf{G})$ and 1 cohort of liposarcoma patients $(\mathbf{H})$ based on analyses of data in the PrognoScan database. Corrected $P$ values are shown.

Our results were independent of the in vivo metastasis assay methods used, as these results were reproduced using various well-established experimental models of metastasis, including those employing tail vein or intrasplenic injection; spontaneous primary orthotopic metastasis assays (37); and mouse cancer cells in immunocompetent mice and human cancer cells in immunodeficient mice. In orthotopic mouse models, PPARD inhibition of metastases far exceeded the inhibition of primary tumor growth in Panc-02 cells 
and was independent of $4 \mathrm{~T} 1$ primary tumor growth. These findings indicated that PPARD in cancer cells impacts metastasis independently of the inhibition of the growth of the primary tumor. Our results were also independent of a particular cell line or clone, as they were verified in various stably transfected cell lines and clones. PPARD's effects on metastasis were observed with various cancer cell models, including those employing melanoma cells, colon cancer cells, lung cancer cells, pancreatic cancer cells, and breast cancer cells, which demonstrates that PPARD's effects were not specific to a particular type of cancer. Furthermore, our findings were not secondary to the off-target effects of RNA inhibition, as they were reproduced not only with different shRNA constructs targeting different PPARD sequences but also with a well-characterized model of PPARD genetic deletion via homologous recombination in HCT116 cells (23); moreover, the reconstitution of PPARD in this model restored the cells' metastatic capacity. In addition, PPARD ligand activation by a pharmaceutical agonist enhanced metastasis formation without the genetic manipulation of PPARD expression. Collectively, these findings underscore the critical effects that PPARD expression in cancer cells have on metastasis.

Our mechanistic studies of PPARD expression modulation in cancer cells revealed that PPARD strongly enhanced key mechanisms in metastasis, including angiogenesis, EMT, invasion, and migration. Previous studies have reported contradictory data regarding the effects of PPARD on VEGF expression and angiogenesis in cancer cells $(11,13-16)$. In the present study, we showed that PPARD in cancer cells upregulated VEGF expression and promoted angiogenesis. We also found that among non-VEGF pro-angiogenesis mechanisms, which are important because tumors invariably become resistant to VEGF inhibition (38, 39), PPARD targets IL-8. IL-8 is a chemokine overexpressed in various human cancers that strongly promotes tumor angiogenesis and metastasis (40-43). PPARD agonists have been reported to increase IL-8 expression in noncancerous endothelial cells (44). During our preparation of this article, an independent group reported that PPARD deletion in HCT116 cells inhibited IL-8 expression and angiogenesis in vitro (45). However, another group reported that PPARD agonists inhibited IL-8 in human monocyte-derived macrophages in a global network screen; these findings were not confirmed in human WPMY-1 myofibroblastic or MDA-MB-231 breast cancer cell lines (46). The inconsistency in these studies' findings regarding the link between PPARD and IL-8 may reflect inherent differences between the various in vitro experiments the studies employed. In contrast, our findings from in vitro mechanistic studies, in vivo preclinical metastasis studies, and human studies that included patients consistently establish a role for PPARD in the regulation of IL-8 in cancer cells.

Our comprehensive transcriptome analysis comparing HCT116 cells with and HCT116 cells without genetic deletion of PPARD identified differentially expressed genes involved in invasion and metastasis. Approximately two-thirds of these differentially expressed genes were downregulated by PPARD deletion, which indicates that PPARD in cancer cells functions predominately as a transcriptional activator. The top functions Ingenuity Pathway Analysis identified for the differentially expressed genes — "cancer," "cellular growth and proliferation," and "cell movement" - support the concept that PPARD promotes pro-metastatic functions. The clinical relevance of several novel PPARD pro-metastatic targets among the differentially expressed genes was supported by their association with PPARD expression in a large TCGA dataset of colorectal cancer patients. This gene list included IL-8, which validated our earlier finding of PPARD's regulatory function for IL-8, and NRG1, which we reported previously to be upregulated by PPARD in a genetic PPARD overexpression mouse model (34).

We also found that for the first time to our knowledge that PPARD regulated several pro-metastatic targets: GJA1, SPARC, STC1, VIM, and SNCG. The GJA1 gene encodes connexin43 (CX43), a transmembrane protein linked to enabling tumor cell extravasation and metastasis (47-49). The matricellular protein SPARC is a breast cancer metastasis signature gene (50) that contributes to altering extracellular matrix remodeling to promote metastasis (51). SPARC promotes tumor progression and increases colorectal cancer recurrence risk (5254). STC1 is a secreted glycoprotein that modulates angiogenesis and promotes tumorigenesis and metastasis in various cancers, including breast (55), lung (56), and colorectal cancer (57). SNCG is a small soluble protein that interferes with the normal mitotic checkpoint and forms a positive feedback loop with insulin-like growth factor signaling (58). SNCG promotes tumor invasion, metastasis, and resistance to antineoplastic-induced apoptosis (58-60). SNCG expression is upregulated in several common human cancers (e.g., lung, breast, prostate, colon) and is correlated with metastasis risk (61-63).

Our findings also indicate that PPARD expression in cancer cells strongly promoted EMT. VIM is a strong marker of EMT and mechanistically contributes to promoting EMT, tumor progression, and metastasis $(64,65)$. PPARD KO in HCT-116 cells markedly reduced VIM (>32-fold). The clinical relevance of the link 


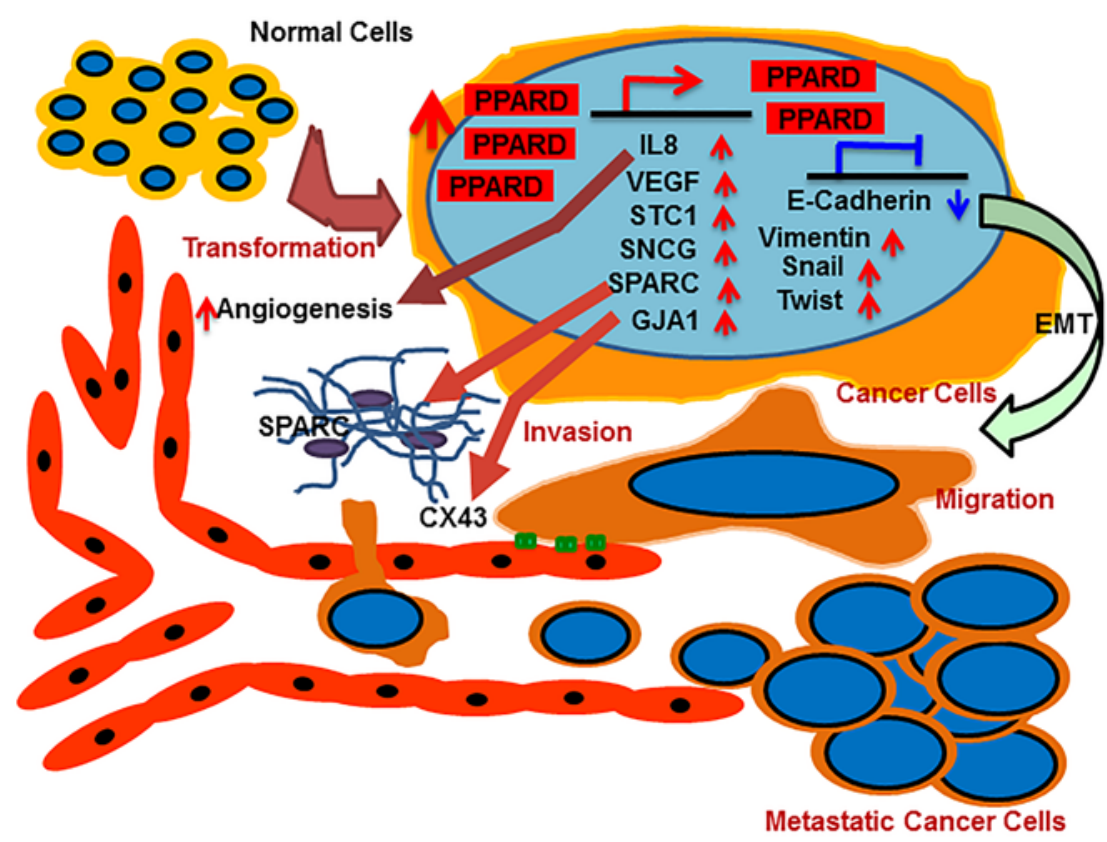

Figure 13. Conceptual scheme of the mechanisms by which PPARD upregulation in cancer cells promotes metastasis. PPARD is upregulated in cancer cells. The upregulated PPARD in turn transcriptionally increases the expression of various pro-metastatic genes (e.g., IL8, VECF, STC1, VIM, SPARC), which promote EMT and other important metastatic events.

between PPARD and vimentin was further supported in analyses of large TCGA databases of patients with colorectal adenocarcinoma, breast cancer, lung cancer, and prostate cancer. PPARD also downregulated E-cadherin and GRHL2 and upregulated ZEB1, SNAIL, and TWIST, which are important transcriptional regulators of EMT (64). At the time of this writing, only 2 other studies have linked PPARD to EMT: In one study, PPARD was reported to promote EMT by enhancing SRC signaling to drive ultraviolet light-driven skin tumorigenesis (22), and in another study, PPARD agonists were reported to

upregulate SNAIL expression in melanoma cells, enhancing their migration and invasion in vitro (21). Previous studies have also shown that PPARD downregulation by siRNA in PC3 prostate cancer cells inhibited cell migration and matrix metalloproteinase-9 and caveolin 1 expression in vitro (66) and that pharmaceutical inhibition of PPARD suppressed MDA-MB-231 breast cancer cell invasion via the downregulation of TGF- $\beta$ angiopoietin-like 4 signaling (20). Although these previous studies' in vitro findings support PPARD's pro-metastatic role in cancer, the present study's findings provide mechanistic insights into how PPARD expression in cancer cells regulates cancer cell invasion, migration, and metastasis that are supported with in vivo metastasis modeling and whose translational relevance is established with data sets from large patient cohorts.

Finally, we found that PPARD expression in cancer cells strongly influenced metastasis-free survival in various large patient cohorts. Several groups have reported that PPARD is upregulated in various human cancers, including colorectal, pancreatic, and lung cancer $(5-8,67)$, but others have reported contradictory findings (68). The present study's findings show that PPARD was upregulated in colorectal tumors in one of the largest cohorts of colorectal cancer patients yet studied. In addition, PPARD was expressed in both the cytoplasm and nuclei of cancer cells but in only the nuclei of normal cells. The unexpected mislocalization of this nuclear protein during colorectal tumorigenesis is in agreement with a prior report (67). While PPARD mislocalization might be secondary to the protein overexpression in cancer cells, future studies to determine its biological significance are warranted. More importantly, we found that high PPARD expression in primary tumors was associated with significantly shorter metastasis-free survival in our cohort of patients with stage III colorectal cancer. These findings were confirmed in analyses of independent datasets from patients with other cancers (breast cancer, lung cancer, and liposarcoma). We also found that high PPARD or IL-8 expression had parallel association with poor prognosis in various cancer patients. The IL-8 findings are in agreement with those of previous studies showing its association with poor prognosis in cancer patients (69-71). Prior studies of PPARD in humans have reported mixed results. In one report, high PPARD expression levels in human cancers were correlated with advanced stages and metastases (7), but another study indicated that PPARD expression levels in lymph node metastases from rectal cancer were lower than those in paired primary tumors (72). Our newly reported findings in various cancer patient cohorts, including the largest reported cohort of 1,609 breast cancer patients, show that PPARD expression is associated with an increased risk of metastasis and reduced metastasis-free survival.

In conclusion, our findings identify PPARD expression in cancer cells as a critical driver of metastasis (Figure 13). They also argue against the hypothesis that PPARD has different effects on tumorigenesis depending on whether it is expressed in cancer cells or non-cancer cells. The improved understanding of PPARD's role in metastasis that our findings impart is necessary for guiding the future development of small molecules to inhibit PPARD and suppress metastasis. 


\section{Methods}

Generation of cell lines with stable transduction of PPARD-shRNA. Plasmid transfections: Four PPARDshRNA plasmids against mouse PPARD in pGFP-V-RS vector were purchased from Origene. The PPARD shRNA sequences were as follows: shRNA-A, AGGTAGAAGCCATCCAGGACACCATTCTG; shRNA-B, CGTGTTCTACCGCTGCCAGTCCACCACAG; shRNA-C, TCAGGCGGCAGCCTCAACATGGAATGTCG; shRNA-D, AGCATCCTCACCGGCAAGTCCAGCCACAA. The shRNA pGFP-V-RS plasmid was provided by Origene as a negative control. B16-F10, LLC-GFP, and Panc-02 cells were transiently transfected with these 4 PPARD shRNAs and the control plasmid using Lipofectamine 2000 (Life Technologies) for 48 hours, and the PPARD shRNA plasmids that reduced PPARD mRNA by $70 \%$ or more compared with the control plasmid, according to qRT-PCR, were used to establish stable cell lines. Cells transfected with PPARD-shRNA-A (B16-F10 and Panc-02 cells) or PPARD-shRNA-B (LLC-GFP cells) were selected with puromycin $(3 \mu \mathrm{g} / \mathrm{ml})$. Clones with stable transfection were isolated and expanded.

Lentivirus transduction: Two GIPZ PPARD shRNA lentiviral plasmids (clone ID V3LMM_426066 and V2LMM_244811) and GIPZ non-silencing lentiviral shRNA control plasmid (catalog RHS4346) were all purchased from GE Dharmacon and were packaged into lentivirus particles (PPARD-shRNA-C or -D or control-shRNA) in the MD Anderson Cancer Center's shRNA and ORFeome Core Facility. B16-F10, LLC-GFP, and 4T1 cells were transduced with either PPARD-shRNA-C or -D or control-shRNA lentiviral particles (MOI of 10 for all lentiviruses) with hexadimethrine bromide $(8 \mu \mathrm{g} / \mathrm{ml})$. The cells were selected with puromycin ( $3 \mu \mathrm{g} / \mathrm{ml}$ for both B16-F10 and LLC-GFP and $6 \mu \mathrm{g} / \mathrm{ml}$ for $4 \mathrm{~T} 1$ cells) 48 hours after transduction. Clones with stable transduction were isolated and expanded.

Syngeneic lung tumor metastasis models. To determine the effect of PPARD on tumor growth in vivo, we assessed the formation of lung metastases after injecting stable PPARD-shRNA B16-F10 or LLC-GFP clones into the tail veins of mice. PPARD-shRNA B16-F10 clones $\left(1 \times 10^{5}\right.$ viable cells in $200 \mu 1 \mathrm{DMEM}$ with high glucose), LLC-GFP clones $\left(1 \times 10^{6}\right.$ viable cells in $200 \mu 1$ DMEM with high glucose), or control-shRNA clones were injected into the tail veins of 6- to 8-week-old B6 mice maintained under specific pathogen-free conditions. Three weeks (B16-F10) or 2 weeks (LLC-GFP) after injection, the mice were killed by $\mathrm{CO}_{2}$ asphyxiation, the lung tumors were counted, and lung images were captured. Tissue specimens from each group were snap frozen, preserved in RNAlater (Life Technologies), and fixed in formalin and embedded in paraffin for histologic examination or embedded in optimum cutting temperature compound for frozen tissue sectioning.

Lung metastasis xenograft formation by human colon cancer cells in immunodeficient mice. Female athymic nude mice, 6-8 weeks old, were purchased from MD Anderson Cancer Center's Animal Experimental Radiation Oncology Animal Facility and maintained under specific pathogen-free conditions. The mice were inoculated with one of the HCT116 clones (15-18 mice per group). In brief, $1.0 \times 10^{6} \mathrm{HCT} 116$ parental cells (WT), PPARD-KO cells, or PPARD-KO cells stably transfected with PPARD vector (PPARD-KOPD) or control vector (PPARD-KO-C) in $200 \mu \mathrm{DMEM}$ with high glucose were injected into the tail vein of each mouse. Six weeks after injection, the mice were killed by $\mathrm{CO}_{2}$ asphyxiation, the lung tumors were counted, and lung images were captured. Tumor specimens from each group were snap frozen, preserved in RNAlater (Life Technologies), or fixed in formalin.

RNA sequencing for transcriptome analyses. Illumina-compatible mRNA libraries were constructed using the TruSeq RNA sample prep kit v2 (Illumina Inc.). Five hundred nanograms of total RNA were enriched for Poly-A mRNA using Oligo-dT magnetic beads. The resulting Poly-A mRNA was fragmented to a median size of $150 \mathrm{bp}$ using divalent cations. Randomly primed first- and second-strand synthesis was then performed to create double-stranded cDNA. The ends of the double-stranded cDNA fragments were repaired, 5'-phosphorylated, and 3'-A tailed, and then the Illumina-specific Y-shaped indexed adapters were ligated. The adaptor-ligated fragments were then purified and enriched by 7 cycles of PCR amplification. The amplified library was then quantified by qPCR using a library quantification kit (Kapa Biosystems) and assessed for size distribution using the Bioanalyzer system (Agilent Technologies). Eight libraries were multiplexed and sequenced on an Illumina HiSeq2000 instrument using the 75-bp paired-end format. After sequencing, BCL files were converted into ".fastq.gz" files, and individual sample libraries were de-multiplexed using CASAVA 1.8.2 (Illumina Inc.) with no mismatches. The RNA sequencing data have been deposited into the NCBI's Gene Expression Omnibus (GSE89729). 
Study approval. All reported experiments related to human subjects were pre-approved by The University of Texas MD Anderson Cancer Center Institutional Review Board. All animal experiments were pre-approved by the MD Anderson Cancer Center Institutional Animal Care and Use Committee.

Statistics. Comparisons of single-factor experimental conditions for continuous outcome measures were performed using 1-way ANOVA, and Bonferroni adjustments were used for all multiple comparisons. Twoway analysis of variance was used to analyze data involving the simultaneous consideration of two factors. Poisson regression was used for tumor count analyses as described previously (15). The data were log-transformed as necessary to accommodate the normality and homoscedasticity assumptions implicit to the statistical procedures used. All tests were 2-sided and conducted at a significance level of $P<0.05$. Data were analyzed using SAS software (SAS Institute). The analysis of distant metastasis-free survival included patients with stage III colorectal cancer with at least 120 days of distant metastasis-free survival after surgery to exclude misclassified cases. The log-transformed ratio of immunohistochemistry scores (log score ratio) of cytoplasmic to nuclear staining of tumor tissue was calculated for each case. Cases were grouped as having score ratios below the median log score ratio (low PPARD expression) or at or above the median log score ratio (high PPARD expression), and metastasis-free survival rates as a function of time were estimated using the Kaplan-Meier method with Prism 6.0 software (GraphPad Software). The difference in distant metastasis-free survival between patients with high and low PPARD expression was analyzed using the log-rank test.

Additional experimental details are included in Supplemental Methods.

\section{Author contributions}

IS developed the conceptual work. IS and X Zuo designed experiments, analyzed the data and wrote the paper. X Zuo, WX, MX, RT, MJM, and FM performed experiments. X Zheng and JW performed bioinformatics analyses, and JSM supervised and contributed to statistical analyses. WX, MG, AR, and RB performed pathology analyses. CE, SK, and DMM provided clinical and pathological data and human clinical samples. AKS guided in vivo angiogenesis studies and provided conceptual feedback. DW and $\mathrm{MCH}$ provided conceptual feedback.

\section{Acknowledgments}

Funding for this research was provided by the National Cancer Institute (R01-CA142969 to IS and Cancer Center Support Grant CA016672) and the Cancer Prevention and Research Institute of Texas (RP140224 to IS). We thank Béatrice Desvergne for providing PPARD germline KO mice and Keiping Xie for providing IL-8 promoter luciferase constructs. We thank Yaying Yang for technical support.

Address correspondence to: Imad Shureiqi, Department of Gastrointestinal Medical Oncology, The University of Texas MD Anderson Cancer Center, 1515 Holcombe Boulevard, Houston, Texas 77030-4009, USA. Phone: 713.792.2828; E-mail: ishureiqi@mdanderson.org.

1. Fidler IJ. The pathogenesis of cancer metastasis: the 'seed and soil' hypothesis revisited. Nat Rev Cancer. 2003;3(6):453-458.

2. Mehlen P, Puisieux A. Metastasis: a question of life or death. Nat Rev Cancer. 2006;6(6):449-458.

3. Sethi N, Kang Y. Unravelling the complexity of metastasis - molecular understanding and targeted therapies. Nat Rev Cancer. 2011;11(10):735-748.

4. Xu M, Zuo X, Shureiqi I. Targeting peroxisome proliferator-activated receptor- $\beta / \delta$ in colon cancer: how to aim? Biochem Pharmacol. 2013;85(5):607-611.

5. He TC, Chan TA, Vogelstein B, Kinzler KW. PPARdelta is an APC-regulated target of nonsteroidal anti-inflammatory drugs. Cell. 1999;99(3):335-345.

6. Gupta RA, et al. Prostacyclin-mediated activation of peroxisome proliferator-activated receptor delta in colorectal cancer. Proc Natl Acad Sci USA. 2000;97(24):13275-13280.

7. Abdollahi A, et al. Transcriptional network governing the angiogenic switch in human pancreatic cancer. Proc Natl Acad Sci USA. 2007;104(31):12890-12895.

8. Pedchenko TV, Gonzalez AL, Wang D, DuBois RN, Massion PP. Peroxisome proliferator-activated receptor beta/delta expression and activation in lung cancer. Am J Respir Cell Mol Biol. 2008;39(6):689-696.

9. Peters JM, Shah YM, Gonzalez FJ. The role of peroxisome proliferator-activated receptors in carcinogenesis and chemoprevention. Nat Rev Cancer. 2012;12(3):181-195.

10. Harman FS, Nicol CJ, Marin HE, Ward JM, Gonzalez FJ, Peters JM. Peroxisome proliferator-activated receptor-delta attenuates colon carcinogenesis. Nat Med. 2004;10(5):481-483.

11. Wang D, et al. Crosstalk between peroxisome proliferator-activated receptor delta and VEGF stimulates cancer progression. Proc Natl Acad Sci USA. 2006;103(50):19069-19074. 
12. Coleman JD, Thompson JT, Smith RW, Prokopczyk B, Vanden Heuvel JP. Role of Peroxisome Proliferator-Activated Receptor $\beta / \delta$ and B-Cell Lymphoma-6 in Regulation of Genes Involved in Metastasis and Migration in Pancreatic Cancer Cells. PPAR Res. 2013;2013:121956.

13. Fauconnet $\mathrm{S}$, et al. Differential regulation of vascular endothelial growth factor expression by peroxisome proliferator-activated receptors in bladder cancer cells. J Biol Chem. 2002;277(26):23534-23543.

14. Stephen RL, et al. Activation of peroxisome proliferator-activated receptor delta stimulates the proliferation of human breast and prostate cancer cell lines. Cancer Res. 2004;64(9):3162-3170.

15. Zuo X, et al. Targeted genetic disruption of peroxisome proliferator-activated receptor-delta and colonic tumorigenesis. $J$ Natl Cancer Inst. 2009;101(10):762-767.

16. Hollingshead HE, et al. Peroxisome proliferator-activated receptor-beta/delta (PPARbeta/delta) ligands do not potentiate growth of human cancer cell lines. Carcinogenesis. 2007;28(12):2641-2649.

17. Folkman J. Role of angiogenesis in tumor growth and metastasis. Semin Oncol. 2002;29(6 Suppl 16):15-18.

18. Hanahan D, Weinberg RA. Hallmarks of cancer: the next generation. Cell. 2011;144(5):646-674.

19. Müller-Brüsselbach S, et al. Deregulation of tumor angiogenesis and blockade of tumor growth in PPARbeta-deficient mice. EMBO J. 2007;26(15):3686-3698.

20. Adhikary T, et al. Inverse PPAR $\beta / \delta$ agonists suppress oncogenic signaling to the ANGPTL 4 gene and inhibit cancer cell invasion. Oncogene. 2013;32(44):5241-5252.

21. Ham SA, et al. Ligand-activated PPAR $\delta$ modulates the migration and invasion of melanoma cells by regulating Snail expression. Am J Cancer Res. 2014;4(6):674-682.

22. Montagner A, et al. Src is activated by the nuclear receptor peroxisome proliferator-activated receptor $\beta / \delta$ in ultraviolet radiation-induced skin cancer. EMBO Mol Med. 2014;6(1):80-98.

23. Park BH, Vogelstein B, Kinzler KW. Genetic disruption of PPARdelta decreases the tumorigenicity of human colon cancer cells. Proc Natl Acad Sci USA. 2001;98(5):2598-2603.

24. Gray MJ, et al. Therapeutic targeting of neuropilin-2 on colorectal carcinoma cells implanted in the murine liver. $J$ Natl Cancer Inst. 2008;100(2):109-120

25. Schneider C, et al. Tumour suppression induced by the macrophage activating lipopeptide MALP-2 in an ultrasound guided pancreatic carcinoma mouse model. Gut. 2004;53(3):355-361.

26. Aslakson CJ, Miller FR. Selective events in the metastatic process defined by analysis of the sequential dissemination of subpopulations of a mouse mammary tumor. Cancer Res. 1992;52(6):1399-1405.

27. Jiang Y, et al. A Sucrose-Enriched Diet Promotes Tumorigenesis in Mammary Gland in Part through the 12-Lipoxygenase Pathway. Cancer Res. 2016;76(1):24-29.

28. Barak Y, et al. Effects of peroxisome proliferator-activated receptor delta on placentation, adiposity, and colorectal cancer. Proc Natl Acad Sci USA. 2002;99(1):303-308.

29. Nadra K, et al. Differentiation of trophoblast giant cells and their metabolic functions are dependent on peroxisome proliferator-activated receptor beta/delta. Mol Cell Biol. 2006;26(8):3266-3281.

30. Ito Y, et al. Inhibition of angiogenesis and vascular leakiness by angiopoietin-related protein 4. Cancer Res. 2003;63(20):6651-6657.

31. Opitz L, et al. Impact of RNA degradation on gene expression profiling. BMC Med Genomics. 2010;3:36.

32. Cerami E, et al. The cBio cancer genomics portal: an open platform for exploring multidimensional cancer genomics data. Cancer Discov. 2012;2(5):401-404.

33. Gao J, et al. Integrative analysis of complex cancer genomics and clinical profiles using the cBioPortal. Sci Signal. 2013;6(269):p11.

34. Zuo X, et al. Potentiation of colon cancer susceptibility in mice by colonic epithelial PPAR- $\delta / \beta$ overexpression. $J$ Natl Cancer Inst. 2014;106(4):dju052.

35. Mizuno H, Kitada K, Nakai K, Sarai A. PrognoScan: a new database for meta-analysis of the prognostic value of genes. $B M C$ Med Genomics. 2009;2:18.

36. Györffy B, et al. An online survival analysis tool to rapidly assess the effect of 22,277 genes on breast cancer prognosis using microarray data of 1,809 patients. Breast Cancer Res Treat. 2010;123(3):725-731.

37. Khanna C, Hunter K. Modeling metastasis in vivo. Carcinogenesis. 2005;26(3):513-523.

38. Ellis LM, Hicklin DJ. Pathways mediating resistance to vascular endothelial growth factor-targeted therapy. Clin Cancer Res. 2008;14(20):6371-6375.

39. Bergers G, Hanahan D. Modes of resistance to anti-angiogenic therapy. Nat Rev Cancer. 2008;8(8):592-603.

40. Waugh DJ, Wilson C. The interleukin-8 pathway in cancer. Clin Cancer Res. 2008;14(21):6735-6741.

41. Kim MY, et al. Tumor self-seeding by circulating cancer cells. Cell. 2009;139(7):1315-1326.

42. Korkaya H, Liu S, Wicha MS. Regulation of cancer stem cells by cytokine networks: attacking cancer's inflammatory roots. Clin Cancer Res. 2011;17(19):6125-6129.

43. Ning Y, et al. Interleukin-8 is associated with proliferation, migration, angiogenesis and chemosensitivity in vitro and in vivo in colon cancer cell line models. Int J Cancer. 2011;128(9):2038-2049.

44. Meissner M, et al. Peroxisome proliferator-activated receptor \{delta\} activators induce IL-8 expression in nonstimulated endothelial cells in a transcriptional and posttranscriptional manner. J Biol Chem. 2010;285(44):33797-33804.

45. Jeong E, Koo JE, Yeon SH, Kwak MK, Hwang DH, Lee JY. PPAR $\delta$ deficiency disrupts hypoxia-mediated tumorigenic potential of colon cancer cells. Mol Carcinog. 2014;53(11):926-937.

46. Adhikary T, et al. The transcriptional PPAR $\beta / \delta$ network in human macrophages defines a unique agonist-induced activation state. Nucleic Acids Res. 2015;43(10):5033-5051.

47. Villares GJ, Zigler M, Bar-Eli M. The emerging role of the thrombin receptor (PAR-1) in melanoma metastasis--a possible therapeutic target. Oncotarget. 2011;2(1-2):8-17.

48. Dubina MV, Iatckii NA, Popov DE, Vasil'ev SV, Krutovskikh VA. Connexin 43, but not connexin 32, is mutated at advanced stages of human sporadic colon cancer. Oncogene. 2002;21(32):4992-4996. 
49. Ryszawy D, et al. Functional links between Snail-1 and Cx43 account for the recruitment of Cx43-positive cells into the invasive front of prostate cancer. Carcinogenesis. 2014;35(9):1920-1930.

50. Minn AJ, et al. Genes that mediate breast cancer metastasis to lung. Nature. 2005;436(7050):518-524.

51. Framson PE, Sage EH. SPARC and tumor growth: where the seed meets the soil? J Cell Biochem. 2004;92(4):679-690.

52. Ribeiro N, Sousa SR, Brekken RA, Monteiro FJ. Role of SPARC in bone remodeling and cancer-related bone metastasis. $J$ Cell Biochem. 2014;115(1):17-26.

53. Kaiser S, et al. Transcriptional recapitulation and subversion of embryonic colon development by mouse colon tumor models and human colon cancer. Genome Biol. 2007;8(7):R131.

54. O'Connell MJ, et al. Relationship between tumor gene expression and recurrence in four independent studies of patients with stage II/III colon cancer treated with surgery alone or surgery plus adjuvant fluorouracil plus leucovorin. J Clin Oncol. 2010;28(25):3937-3944.

55. Chang AC, et al. STC1 expression is associated with tumor growth and metastasis in breast cancer. Clin Exp Metastasis. 2015;32(1):15-27.

56. Du YZ, Gu XH, Li L, Gao F. The diagnostic value of circulating stanniocalcin-1 mRNA in non-small cell lung cancer. $J$ Surg Oncol. 2011;104(7):836-840.

57. Peña C, et al. STC1 expression by cancer-associated fibroblasts drives metastasis of colorectal cancer. Cancer Res. 2013;73(4):1287-1297.

58. Li M, et al. The reciprocal regulation of gamma-synuclein and IGF-I receptor expression creates a circuit that modulates IGF-I signaling. J Biol Chem. 2010;285(40):30480-30488.

59. Miao S, et al. Synuclein $\gamma$ compromises spindle assembly checkpoint and renders resistance to antimicrotubule drugs. $\mathrm{Mol}$ Cancer Ther. 2014;13(3):699-713.

60. Ahmad M, Attoub S, Singh MN, Martin FL, El-Agnaf OM. Gamma-synuclein and the progression of cancer. FASEB J 2007;21(13):3419-3430.

61. Liu H, et al. Loss of epigenetic control of synuclein-gamma gene as a molecular indicator of metastasis in a wide range of human cancers. Cancer Res. 2005;65(17):7635-7643.

62. Murff HJ, et al. A prospective study of dietary polyunsaturated fatty acids and colorectal cancer risk in Chinese women. Cancer Epidemiol Biomarkers Prev. 2009;18(8):2283-2291.

63. Hibi T, et al. Synuclein-gamma is closely involved in perineural invasion and distant metastasis in mouse models and is a novel prognostic factor in pancreatic cancer. Clin Cancer Res. 2009;15(8):2864-2871.

64. Satelli A, Li S. Vimentin in cancer and its potential as a molecular target for cancer therapy. Cell Mol Life Sci. 2011;68(18):3033-3046.

65. De Craene B, Berx G. Regulatory networks defining EMT during cancer initiation and progression. Nat Rev Cancer. 2013;13(2):97-110.

66. Her NG, et al. PPAR $\delta$ promotes oncogenic redirection of TGF- $\beta 1$ signaling through the activation of the ABCA1-Cav1 pathway. Cell Cycle. 2013;12(10):1521-1535.

67. Takayama O, et al. Expression of PPARdelta in multistage carcinogenesis of the colorectum: implications of malignant cancer morphology. Br J Cancer. 2006;95(7):889-895.

68. Foreman JE, et al. Functional characterization of peroxisome proliferator-activated receptor- $\beta / \delta$ expression in colon cancer Mol Carcinog. 2011;50(11):884-900

69. Lambrechts D, et al. Evaluation of efficacy and safety markers in a phase II study of metastatic colorectal cancer treated with aflibercept in the first-line setting. Br J Cancer. 2015;113(7):1027-1034.

70. Kopetz S, et al. Phase II trial of infusional fluorouracil, irinotecan, and bevacizumab for metastatic colorectal cancer: efficacy and circulating angiogenic biomarkers associated with therapeutic resistance. J Clin Oncol. 2010;28(3):453-459.

71. Abajo A, et al. Identification of predictive circulating biomarkers of bevacizumab-containing regimen efficacy in pre-treated metastatic colorectal cancer patients. Br J Cancer. 2012;107(2):287-290.

72. Yang L, Zhang H, Zhou ZG, Yan H, Adell G, Sun XF. Biological function and prognostic significance of peroxisome proliferator-activated receptor $\delta$ in rectal cancer. Clin Cancer Res. 2011;17(11):3760-3770. 